\title{
Statistical physical approach to describe the collective properties of dislocations
}

\author{
István Groma \\ Department of General Physics, Eötvös University Budapest, 1117 Budapest Pázmány P. \\ sétány $1 / \mathrm{A}$
}

\section{Introduction}

The concept of dislocation was introduced by Polanyi, Orovan and Taylor in 1934 to explain the almost three orders of magnitude difference between the measured and theoretically estimated flow stress of crystalline materials. During the next 20-30 years, thanks to the contribution of a vast number of scientists, the theory of dislocations was successfully applied to explain several properties of the plastic deformation observed experimentally. Among other things the basic phenomena leading to work and precipitation hardening were understood $[1,2,3]$.

On the other hand TEM investigations revealed that dislocations formed during plastic deformation tend to form different dislocation patterns, like the cell structure (see Figure 1.) developing at unidirectional load [4], or the so called ladder structure (see Figure 2.) developing under cyclic loading [5]. In spite of several attempts (KuhlmannWilsdorf et.al. [6, 7, 8], Holt [9], Walgraef and Aifantis[10, 11, 12], Kratochvil et.al. $[13,14,15])$ proposed to model the pattern formation, we are far from the complete understanding of this typically self organizing phenomena. One of the most striking features of the dislocation patterning, which is a great challenge to model, is the large variety of the patterns observed. The variety manifests itself not just in the "geometry" of the dense dislocation regions but also in several statistical properties of the different dislocation ensembles. It is known for example that cyclic loading can lead to periodic structures with well defined self selected length scale [5], while unidirectional loading often results in fractal like structures which do not have any length scales (Hähner et.al. $[16,17])$. Another interesting feature of the patterning process observed recently by Xray diffraction (Székely et.al.[18, 19]) is that in case of unidirectional loading the relative dislocation density fluctuation $\sigma^{2}$ defined as

$$
\sigma^{2}=V \frac{\int \rho^{2}(\vec{r}) d^{3} r}{\left[\int \rho(\vec{r}) d^{3} r\right]^{2}},
$$

where $\rho(\vec{r})$ is the dislocation density and $\mathrm{V}$ is the crystal volumes, undergoes a sharp maximum as deformation proceeds (see Figure 3 ). The result indicates that during plas- 


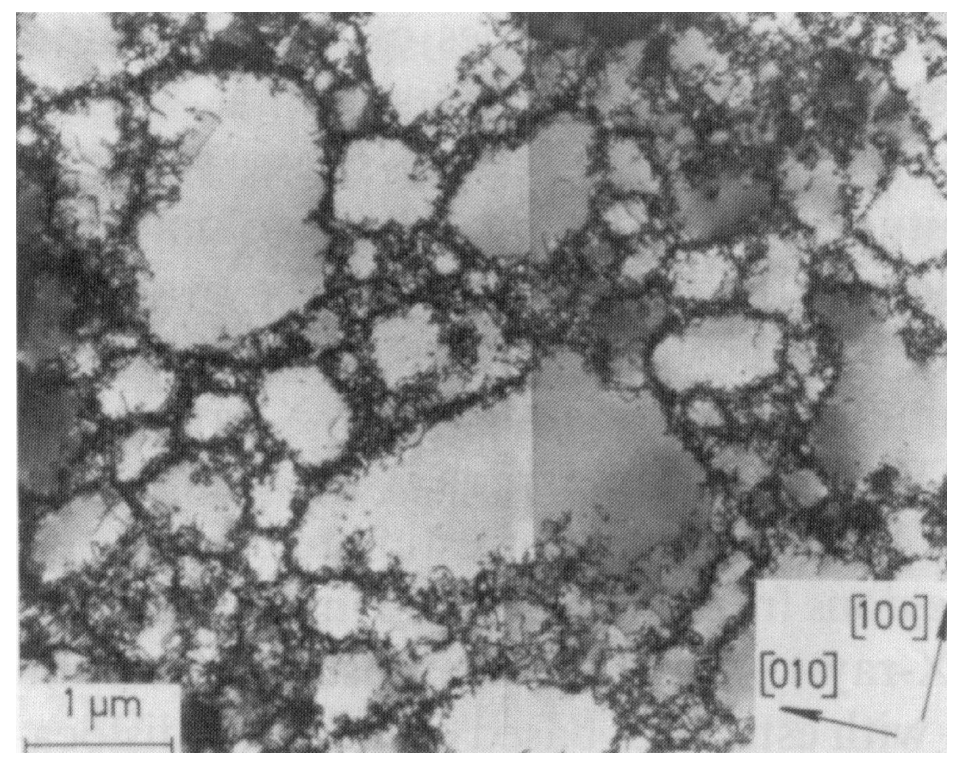

Figure 1. Dislocation cell structure obtained on $\mathrm{Cu}$ single crystal oriented for multiple slip [4].

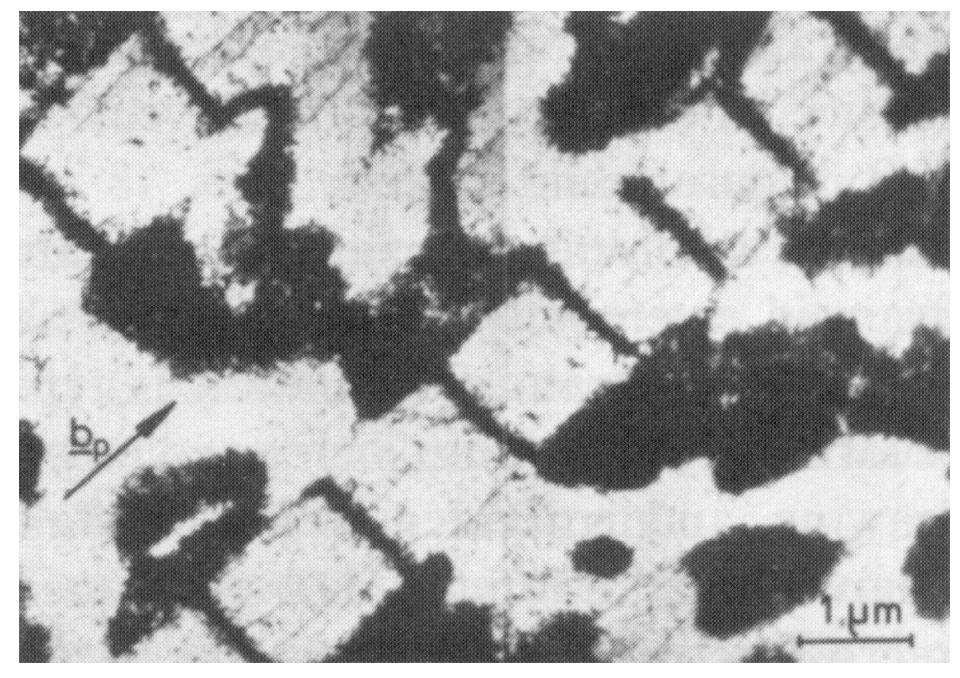

Figure 2. Ladder structure obtained on $\mathrm{Cu}$ single crystal deformed cyclically [5]. 


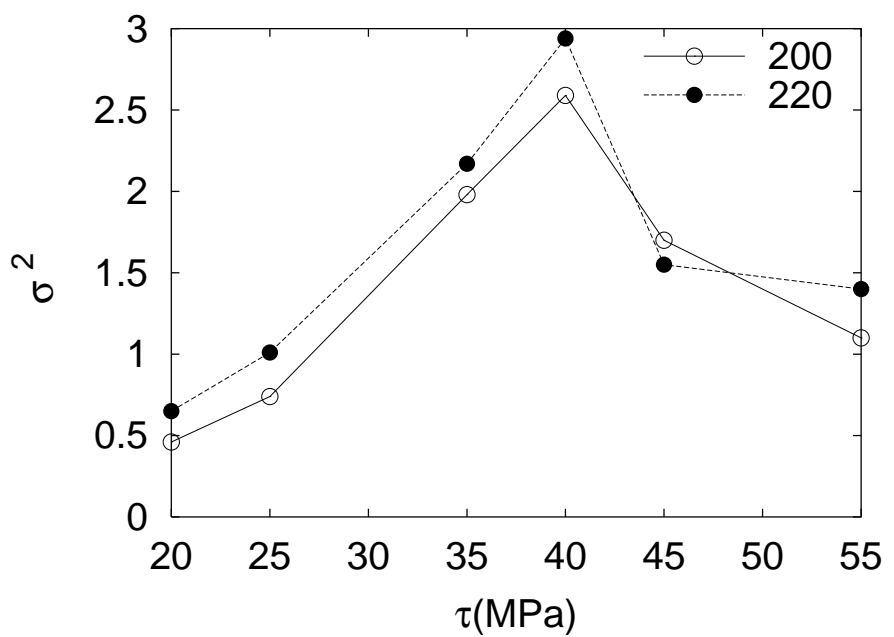

Figure 3. Relative dislocation density fluctuation versus applied stress, obtained on $\mathrm{Cu}$ single crystal oriented for multiple slip. The two curves correspond to relative dislocation density fluctuations determined from the broadening of (200) and (220) Bragg peaks $[18,19]$.

tic deformation the dislocation system tends to become more and more inhomogeneous, but after a certain deformation level (depending on the crystal orientation and temperature ) this separation process cannot continue any longer and the system becomes more and more homogeneous.

Another challenging problem intensively studied nowadays ( Flack et.al.[20], McElhaney et.al. [21]) is that resent experimental investigations revealed (see Figures 4 and 5 ), if the characteristic size of a specimen is less than about $10 \mu \mathrm{m}$ the plastic response of crystalline materials depend on the size. The phenomenon is commonly called "size effect". One can easily explain this size dependence by assuming that the crystalline materials have an internal degree of freedom which "feel" the sample surface. This immediately indicates that a theory able to account for the size effects has to be non-local, since the sample surface is "seen" from the bulk. The simplest possible way to introduce nonlocality is to add gradient terms to the "local" ones. There are several different phenomenological ways to do this. As a possibility one may introduce an effective shear stress $\tau_{\text {eff }}$ as

$$
\tau_{e f f}=\tau(\gamma, \dot{\gamma}, \ldots)+\mu l^{2} \Delta \gamma,
$$

where $\tau(\gamma, \dot{\gamma}, \ldots)$ is the "classical" local stress-strain relation, $\gamma$ is the shear deformation, $\mu$ is the shear modulus and $l$ is a parameter with length dimension. It is important to stress that $l$ has to be introduced for the appropriate dimension of the second term. Since size effects appears at micron scale, the value of $l$ needs to be in the order of $\mu \mathrm{m}$.

During the past 10 years several non-local plasticity theories based on similar arguments explained above have been proposed (Aifantis [22, 23, 24], Flack and Hutchinson 


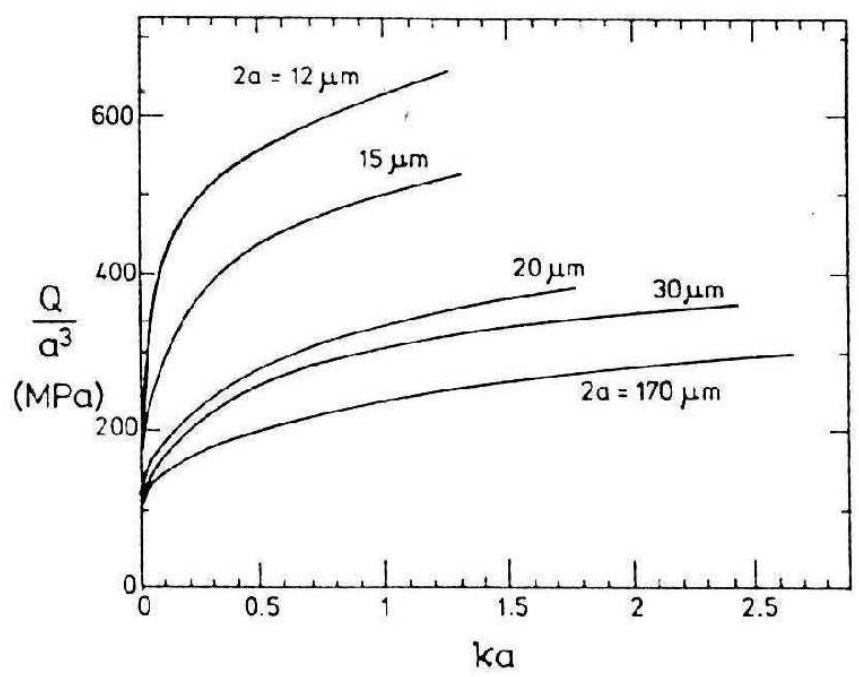

Figure 4. Normalised torque versus shear deformation obtained on torsionally deformed wires with different diameters $a$. The curves indicates, if $a$ is smaller than $50 \mu \mathrm{m}$, the hardening of the wires increase with decreasing diameter [20].

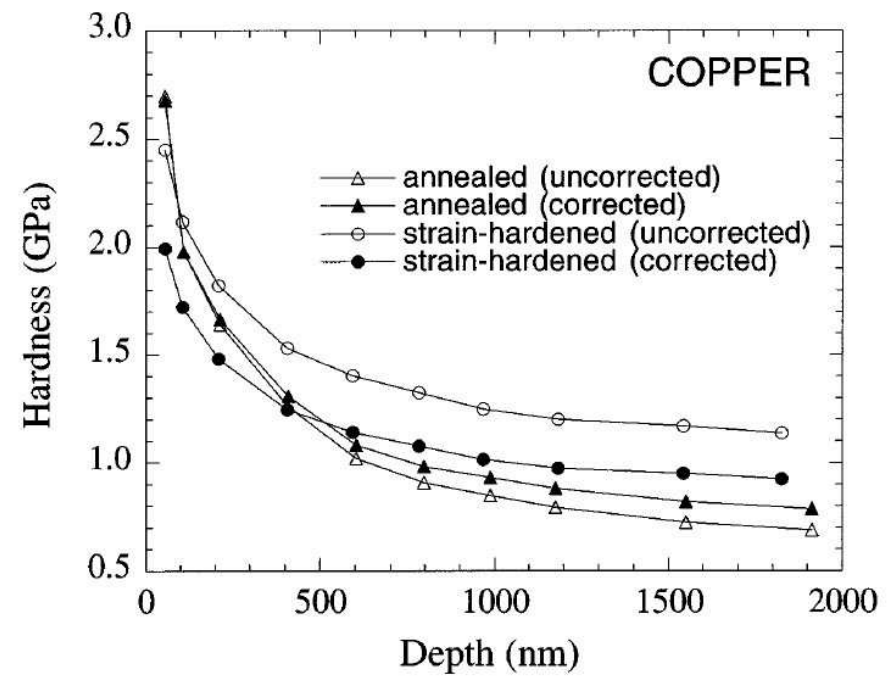

Figure 5. Microhardness versus indentation depth obtained on cold rolled $\mathrm{Cu}$. It can be seen, if the indentation depth is less than $1 \mu \mathrm{m}$ the microhardness increases with decreasing indentation depth [21]. 
[25], Gurtin [26], Svendsen [27]) and successfully applied to explain experimental results. However, these phenomenological non-local theories suffer from the common drawback, namely, the physical origin of the gradient terms are not clear. This is especially pronounced if one tries to explain the origin of a material parameter $(l)$ with a value around $\mu \mathrm{m}$.

On the other hand, we know that in crystalline materials plastic deformation is carried out by dislocations. They definitely act as internal degrees of freedom. Due to the long range stress field of dislocations they "feel" the sample surface. This indicates that size effect is the result of the collective motion of dislocations. The results of discrete dislocation dynamics simulations clearly demonstrate this (see Nicola et.al. [28]).

The aim of the present paper is to explain how the collective behaviour of dislocations can be treated within a statistical physics framework. Since, however, the dislocations form a strongly dissipative system, the classical theory of statistical physics developed for Hamiltonian systems cannot be directly applied. In the first part of the paper the field theory of dislocations developed by Kröner and Kosevich is summarised [29, 30, 31, 32]. Although, the Kröner and Kosevich theory is not able to capture all the statistical features of dislocation systems, it plays an important role in our further considerations.

In the second part of the paper it is shown that for a simple 2D dislocation configuration a continuum theory of dislocations can be rigorously derived. According to numerical investigations it is able to account for certain type of size effects. At the end of this chapter, attempts to generalise the results for 3D are shortly explained.

Finally, in the last part the properties of the internal stress distribution generated by dislocations are discussed.

\section{Kröner-Kosevich field theory of dislocations}

\subsection{Nye's dislocation density tensor}

Shortly after the concept of dislocation was introduced, the elastic field generated by dislocation loops was determined (see for example [1]). In the early 1950s Kröner and Kosevich reconsidered the problem and proposed an extremely elegant formalism to describe the elastic properties of dislocated crystals. In this section the dislocation field theory they have developed is shortly summarised. For more details the reader is refereed to $[29,30,31,32]$.

Let us consider a body subject to shape change. Its deformation is uniquely described by the displacement field $\vec{u}(\vec{r})$. In the small deformation limit the change of atomic distances is measured by the total deformation tensor

$$
\epsilon_{i j}^{t}=\frac{1}{2}\left[\frac{\partial u_{i}}{\partial r_{j}}+\frac{\partial u_{j}}{\partial r_{i}}\right] \quad\left(\hat{\epsilon^{t}}=\left[\frac{d \vec{u}}{d \vec{r}}\right]_{s y m}\right) .
$$

(For better understanding expressions are given both with component and tensor notations. Tensors are denoted by hats.)

In the common theory of elasticity it is assumed that the stress tensor $\hat{\sigma}$ is a unique 
function of the deformation tensor $\hat{\epsilon}^{t}$. In linear elasticity considered in this paper

$$
\sigma_{i j}=L_{i j k l} \epsilon_{l k}^{t} \quad\left(\hat{\sigma}=\hat{L}: \hat{\epsilon}^{t}\right)
$$

where $\hat{L}$ is the tensor of elastic moduli.

Deformation of a body, however, does not necessarily leads to the development of internal stress. If we cut a body into two pieces along a plane, slide the two parts with respect to each other and glue them together, the deformation is obviously nonzero along the plane, but the final stage is stress free. This means, the deformation can have a part which generates stress and another one which does not. According to this, the starting point of the plasticity theory explained below is that the gradient of the displacement field commonly referred as total distortion is the sum of two terms, the so called plastic $\left(\hat{\beta}^{p}\right)$ and elastic $(\hat{\beta})$ distortion i.e.

$$
\frac{\partial u_{j}}{\partial r_{i}}=\beta_{i j}+\beta_{i j}^{p} \quad\left(\frac{d \vec{u}}{d \vec{r}}=\hat{\beta}+\hat{\beta}^{p}\right) .
$$

It needs to be mentioned, that the above expression is valid only in small deformation limit. For large deformations, as a constitutive rule, it is commonly assumed that the final deformation stage of the body is reached by two successive steps, a plastic and an elastic deformation. For small deformations this is equivalent with Eq. (2.3). For the sake of simplicity we consider only this case.

Splitting the total distortion into two terms is rather formal so far. The plastic and elastic distortions need to be defined. It has to be mentioned at this point that, as it is explained in details below, the separation is not unique.

As it is indicated by its name the elastic distortion is that part of the total one, which is related to the stress developed in the body due to the deformation. In linear elasticity

$$
\sigma_{i j}=L_{i j k l} \beta_{l k} \quad(\hat{\sigma}=\hat{L}: \hat{\beta})
$$

However, due to the symmetric properties of $\hat{L}, \hat{\sigma}$ determines only the symmetric part of $\hat{\beta}$. With other words, the stress determines only the elastic deformation $\hat{\epsilon}$ defined as

$$
\epsilon_{i j}=\frac{1}{2}\left(\beta_{i j}+\beta_{j i}\right) \quad\left(\hat{\epsilon}=[\hat{\beta}]_{s y m}\right) .
$$

From Eqs. $(2.4,2.5)$ the elastic deformation reads as

$$
\epsilon_{i j}=L_{i j k l}^{-1} \sigma_{l k} \quad\left(\hat{\epsilon}=\hat{L}^{-1}: \hat{\sigma}\right), .
$$

where $\hat{L}^{-1}$ denotes the inverse of the tensor of elastic moduli.

The next step is to define the plastic distortion. Since, by definition, the total distortion is the gradient of the displacement field, it is curl free, i.e.

$$
e_{i k l} \frac{\partial}{\partial r_{i}} \frac{\partial u_{j}}{\partial r_{i}} \equiv 0 \quad\left(\nabla \times \frac{d \vec{u}}{d \vec{r}} \equiv 0\right)
$$


where $e_{i k l}$ is the antisymmetric tensor. The plastic distortion, however, is not necessarily curl free. After Nye [33] the curl of the plastic distortion is called dislocation density tensor $\hat{\alpha}$ :

$$
\alpha_{i j}=e_{i k l} \frac{\partial}{\partial r_{k}} \beta_{l j}^{p} . \quad\left(\hat{\alpha}=\nabla \times \hat{\beta}^{p}\right)
$$

(In the literature $\hat{\alpha}$ is often refereed to Nye's dislocation density tensor.) From Eqs. $(2.3,2.7,2.8)$ one easily obtains that

$$
\alpha_{i j}=-e_{i k l} \frac{\partial}{\partial r_{k}} \beta_{l j} \quad(\hat{\alpha}=-\nabla \times \hat{\beta}) .
$$

In order to see the physical meaning of $\hat{\alpha}$ introduced above, let us consider its integral for an arbitrary surface $A$

$$
b_{j}=\int_{A} \alpha_{i j} d A_{i}
$$

From the (2.9) expression of $\hat{\alpha}$, with the help of the Stockes' theorem, one can find that

$$
b_{j}=-\int_{A} e_{i k l} \frac{\partial}{\partial r_{k}} \beta_{l j} d A_{i}=-\oint \beta_{i j} d s_{i}=-\oint d u_{i}^{e} .
$$

Equation

$$
b_{j}=-\oint d u_{i}^{e}
$$

obtained is the one Burgers originally used [34] to define a dislocation as a singularity of the elastic displacement field $\vec{u}^{e}$. Therefore, $\hat{\alpha}$ defined by Eq. (2.8) is the net Burgers vector of line singularities crossing a unit area. From Eq. (2.10) one can easily find that for a single straight dislocation

$$
\alpha_{i j}=l_{i} b_{j} \delta^{(2)}(\xi) \quad\left(\hat{\alpha}=\vec{l} \circ \vec{b} \delta^{(2)}(\xi)\right),
$$

where $\vec{l}$ is the tangential vector of the dislocation line and $\xi$ is the distance from the line.

Before we proceed further, it should be mentioned that the dislocation density tensor does not define uniquely the plastic distortion. Knowing $\hat{\alpha}$ leaves $\hat{\beta}^{p}$ uncertain up to a gradient of an arbitrary vector field. However, as it is explained below, this does not affect the stress field created by the dislocation system.

\subsection{Internal stress generated by the dislocation system}

In order to derive the field equations, let us consider the symmetric part of the total distortion. According to Eq. (2.3)

$$
\frac{1}{2}\left(\frac{\partial u_{j}}{\partial r_{i}}+\frac{\partial u_{i}}{\partial r_{j}}\right)=\epsilon_{i j}+\epsilon_{i j}^{p} \quad\left(\left[\frac{d \vec{u}}{d \vec{r}}\right]_{s y m}=\hat{\epsilon}+\hat{\epsilon}^{p}\right),
$$


where $\hat{\epsilon}$ and $\hat{\epsilon}^{p}$ are the symmetric parts of the elastic and plastic distortions, respectively. Using the curl grad $\equiv 0$ identity one can find that

$$
-e_{i k m} e_{j l n} \frac{\partial}{\partial r_{k}} \frac{\partial}{\partial r_{l}}\left(\frac{\partial u_{j}}{\partial r_{i}}+\frac{\partial u_{i}}{\partial r_{j}}\right) \equiv 0 \quad\left(\nabla \times\left[\frac{d \vec{u}}{d \vec{r}}\right]_{s y m} \times \nabla \equiv 0\right) .
$$

It is useful to introduce the tensor operator inc ("incompatibility") defined as

$$
\text { inc }=-e_{i k m} e_{j l n} \frac{\partial}{\partial r_{k}} \frac{\partial}{\partial r_{l}} .
$$

With this definition Eq. (2.15) reads as

$$
\operatorname{inc}\left[\frac{d \vec{u}}{d \vec{r}}\right]_{s y m} \equiv 0 .
$$

For further considerations it is useful to introduce the symmetric tensor field

$$
\eta_{i j}=-e_{i k m} e_{j l n} \frac{\partial}{\partial r_{k}} \frac{\partial}{\partial r_{l}} \epsilon_{m n} \quad(\text { inc } \hat{\epsilon}=\hat{\eta}) .
$$

After a long but straightforward calculation one can obtain from Eqs. $(2.8,2.14,2.17,2.18)$ that

$$
\eta_{i j}=\frac{1}{2}\left(e_{j l n} \frac{\partial}{\partial r_{l}} \alpha_{i m}+e_{i l n} \frac{\partial}{\partial r_{l}} \alpha_{j m}\right) \quad\left(\hat{\eta}=[\hat{\alpha} \times \nabla]_{s y m}\right)
$$

By substituting Eq. (2.6) into (2.18) we arrive at the equation that the stress created by the dislocation has to fulfill:

$$
\eta_{i j}=-e_{i k m} e_{j l n} \frac{\partial}{\partial r_{k}} \frac{\partial}{\partial r_{l}} L_{m n o p}^{-1} \sigma_{o p} \quad\left(\operatorname{inc}\left(\hat{L}^{-1} \hat{\sigma}\right)=\hat{\eta}\right) .
$$

Since, for an arbitrary vector field $\vec{f}$

$$
\operatorname{inc}\left[\frac{d \vec{f}}{d \vec{r}}\right]_{s y m} \equiv 0 .
$$

Eq. (2.20) itself does not determine $\hat{\sigma}$ completely. However, supplementing it with the equilibrium condition

$$
\frac{\partial}{\partial r_{i}} \sigma_{i j}=0 \quad(\operatorname{div} \hat{\sigma}=0)
$$

we already have enough equations to determine the stress field generated by the dislocations. 


\subsection{Second order stress function tensor}

Like in electrodynamics it is useful to reformulate Eqs. (2.20,2.22) into a potential theory. Let us introduce a second order stress function tensor $\hat{\chi}$ defined with the relation

$$
\sigma_{i j}=-e_{i k m} e_{j l n} \frac{\partial}{\partial r_{k}} \frac{\partial}{\partial r_{l}} \chi_{m n} \quad(\hat{\sigma}=\text { inc } \hat{\chi}) .
$$

Due to the identity

$$
\operatorname{div} \text { inc } \equiv 0
$$

the (2.20) form of $\hat{\sigma}$ guarantees that the equilibrium condition (2.22) is fulfilled. With the stress function tensor introduced above Eq. (2.19) reads as

$$
\begin{aligned}
\eta_{i j} & =e_{i k m} e_{j l n} e_{\text {oqv }} e_{\text {puw }} L_{m n o p}^{-1} \frac{\partial}{\partial r_{k}} \frac{\partial}{\partial r_{l}} \frac{\partial}{\partial r_{q}} \frac{\partial}{\partial r_{u}} \chi_{v w} \\
(\hat{\eta} & \left.=\operatorname{inc}\left(\hat{L}^{-1} \operatorname{inc} \hat{\chi}\right)\right) .
\end{aligned}
$$

For an anisotropic medium the above equation is rather difficult to solve, but for isotropic materials, with shear modulus $\mu$ and Poisson's ratio $\nu$, a general solution can be obtained. It is expedient to introduce another tensor potential $\hat{\chi}^{\prime}$ defined as

$$
\begin{aligned}
\chi_{i j}^{\prime} & =\frac{1}{2 \mu}\left(\chi_{i j}-\frac{\nu}{1+2 \nu} \chi_{k k} \delta_{i j}\right) \\
\chi_{i j} & =2 \mu\left(\chi_{i j}^{\prime}+\frac{\nu}{1-\nu} \chi_{k k}^{\prime} \delta_{i j}\right) .
\end{aligned}
$$

By inserting Eq. (2.27) into Eq. (2.25) one can find, if $\hat{\chi}^{\prime}$ fulfills the gauge condition

$$
\frac{\partial}{\partial r_{i}} \chi_{i j}^{\prime}=0 \quad\left(\operatorname{div} \hat{\chi}^{\prime}=0\right)
$$

Eq. (2.23) simplifies to the biharmonic equation

$$
\nabla^{4} \chi_{i j}^{\prime}=\eta_{i j} \quad\left(\nabla^{4} \hat{\chi}^{\prime}=\hat{\eta}\right) .
$$

A remarkable feature of this equation is that the different components of $\hat{\chi}^{\prime}$ obey separate equations making the problem much easier to solve. For an infinite medium the general solution of Eq. (2.29) is

$$
\chi_{i j}^{\prime}(\vec{r})=-\frac{1}{8 \pi} \iiint\left|\vec{r}-\vec{r}^{\prime}\right| \eta_{i j}\left(\vec{r}^{\prime}\right) d V^{\prime}
$$

\section{$2.42 \mathrm{D}$ problems}

In the next section the statistical properties of an ensemble of parallel edge dislocations are discussed. In this case the stress and the strain do not vary along the dislocation line direction $\vec{l}$. Taking $\vec{l}$ parallel to the $z$ axis (with $\vec{l}=(0,0,-1)$ in the above expressions the 
derivatives with respect to $z$ vanish $(\partial / \partial z \equiv 0)$. One can find that Eq. (2.23) simplifies to [30]:

$$
\begin{gathered}
\sigma_{11}=-\frac{\partial^{2} \chi}{\partial y^{2}}, \quad \sigma_{22}=-\frac{\partial^{2} \chi}{\partial x^{2}}, \quad \sigma_{12}=\frac{\partial^{2} \chi}{\partial x \partial y}, \quad \chi \equiv \chi_{33} \\
\sigma_{23}=-\frac{\partial \phi}{\partial x}, \quad \sigma_{13}=\frac{\partial \phi}{\partial y}, \quad \phi=-\frac{\partial \chi_{23}}{\partial x}+\frac{\partial \chi_{31}}{\partial y} .
\end{gathered}
$$

Furthermore, from Eqs. $(2.19,2.25)$ one obtains that the two scalar fields $\chi$ and $\phi$ introduced above obey the equations

$$
\begin{gathered}
\nabla^{4} \chi=\frac{2 \mu}{1-\nu}\left(b_{1} \frac{\partial}{\partial y}-b_{2} \frac{\partial}{\partial x}\right)\left(\rho_{d+}-\rho_{d-}\right) \\
\nabla^{2} \phi=\mu b_{3}\left(\rho_{d+}-\rho_{d-}\right)
\end{gathered}
$$

where $b_{1}, b_{2}$ and $b_{2}$ are the $x, y$, and $z$ directional components of the Burgers vector, respectively. The notations $\rho_{d+}$ and $\rho_{d-}$ stand for the dislocation densities with positive and negative signs, respectively, They are the sum of $\delta\left(\vec{r}-\vec{r}_{i}\right)$ Dirac delta functions, were $\vec{r}_{i}$ denotes the position of a dislocation. For the sake of simplicity we assumed that all dislocations belong to the same slip system (single slip), but the expressions can be easily generalised for multiple slip.

For an infinite medium the solutions of Eqs. $(2.33,2.34)$ read as

$$
\chi(\vec{r})=\frac{2 \mu}{2 \pi(1-\nu)} \int\left(b_{1} \frac{\partial}{\partial y^{\prime}}-b_{2} \frac{\partial}{\partial x^{\prime}}\right)\left[\rho_{d+}\left(\vec{r}^{\prime}\right)-\rho_{d-}\left(\vec{r}^{\prime}\right)\right] R^{2} \ln R d^{2} \vec{r}^{\prime}
$$

and

$$
\phi(\vec{r})=-\frac{\mu b_{3}}{2 \pi} \int\left[\rho_{d+}\left(\vec{r}^{\prime}\right)-\rho_{d-}\left(\vec{r}^{\prime}\right)\right] \ln (R) d^{2} \vec{r}^{\prime}
$$

where $R=\left|\vec{r}-\vec{r}^{\prime}\right|$.

\subsection{Time evolution of the dislocation density tensor}

As it is explained above, if we know the dislocation density tensor (i.e. we know the dislocation line geometry) the internal stress field can be determined from Eq. (2.25). This is however, only a "static" description. In order to be able to describe the response of the dislocation system to external signals, the governing equations of the time evolution of the dislocation density tensor should be determined.

If we take the partial time derivative (denoted by " . ") of Eq. (2.8), we find that

$$
\dot{\alpha}_{i j}+e_{i k l} \frac{\partial}{\partial r_{k}} j_{l j}=0 \quad(\dot{\hat{\alpha}}+\nabla \times \hat{j}=0)
$$


where

$$
\hat{j}=-\dot{\hat{\beta}}^{p}
$$

is called dislocation current density [31]. The above equation is the "conservation law of the Burgers vector" in differential form. Indeed, if we integrate both sides of Eq. (2.37) for an arbitrary area contoured by the closed curve $L$, according to Eq. (2.10), we obtain that

$$
\frac{d b_{j}}{d t}=-\oint_{L} j_{i j} d s_{i}
$$

It is obvious from this relation that $\hat{j}$ is the Burgers vector carried by the dislocations crossing a unit length part of the contour line $L$ per unit time.

For an individual dislocation one can find that

$$
j_{i k}=e_{i l m} l_{l} v_{m} b_{k} \delta^{(2)}(\xi) \quad\left(\hat{j}=(\vec{l} \times \vec{v}) \circ \vec{b} \delta^{(2)}(\xi)\right),
$$

where $\vec{v}$ is the velocity of the dislocation line at a given point. It is important to note that if we added the gradient of an arbitrary vector field to $\hat{j}$ given above, this would also satisfy the conservation law (2.37). The problem is obviously related to the nonuniqueness of the plastic distortion discussed earlier. However, expression (2.40) is the only one which is physically meaningful. One expects that there is no plastic current anywhere else but at the dislocation line. Nevertheless, strictly speaking we have to postulate this.

The above results clearly show that $\hat{j}$ has to be considered as an independent quantity. In order to be able to describe the time evolution of the dislocation system we have to set up a constitutive relation which gives how $\hat{j}$ depends on the dislocation density tensor and the external stress. Due to the long range nature of the dislocation-dislocation interaction, the constitutive relation is obviously non-local in $\hat{\alpha}$. Beside this, the constitutive relation has to be able to account for several different "local" phenomena (self loop interaction, junction formation, annihilation etc.) making even more difficult to determine its form.

One possible approach to handle this problem is to set up the constitutive relation from phenomenological considerations. During the past years several phenomenological expressions were proposed and successfully applied for modelling certain phenomena [26] but the problem is far not completely solved.

Another widely used approach to study the time evolution of dislocation systems is discrete dislocation dynamics (DDD) simulation in which the dislocation loops are considered individually. After setting up velocity laws for the dislocation segments the dislocation loop geometry is updated numerically. Describing the actual numerical techniques used in DDD simulations is out of the scope of this paper. The reader can find the details e.g. in $[35,36,37,38,39,40,42,43,44,45]$. Although DDD simulations are extremely important for the better understanding of the collective properties of dislocations, due to their high computational demand their applicability in engineering practice is limited. 


\subsection{Time evolution of the displacement field}

In the previous part we have discussed how the stress field generated by the dislocations can be determined and what can be said in general about the time evolution of the dislocation density tensor. However, in many applications it is important to determine the displacement field $\vec{u}(\vec{r})$, too.

Let us go back to our starting equation (2.3), multiply it with the tensor of elastic moduli $\hat{L}$, and take the div of the equation. Using Eqs. $(2.4,2.22)$ one obtains that

$$
\frac{\partial}{\partial r_{i}} L_{i j k l} \frac{\partial u_{k}}{\partial r_{l}}=\frac{\partial}{\partial r_{i}} L_{i j k l} \beta_{k l}^{p} \quad\left(\operatorname{div} \hat{L} \frac{d \vec{u}}{d \vec{r}}=\operatorname{div} \hat{L} \hat{\beta}^{p}\right) .
$$

This is formally equivalent with the common equilibrium equation of elasticity with body force density

$$
f_{j}=-\frac{\partial}{\partial r_{i}} L_{i j k l} \beta_{k l}^{p} \quad\left(\vec{f}=-\operatorname{div} \hat{L} \hat{\beta}^{p}\right)
$$

Since, as it is explained earlier, the dislocation density tensor does not determine the plastic distortion uniquely, the above equation is not enough to determine the displacement field. Taking, however, the time derivative of Eq. (2.41), with the (2.38) definition of $\hat{j}$, one finds that

$$
\frac{\partial}{\partial r_{i}} L_{i j k l} \frac{\partial \dot{u}_{k}}{\partial r_{l}}=\frac{\partial}{\partial r_{i}} L_{i j k l} j_{k l} \quad\left(\operatorname{div} \hat{L} \frac{d \dot{\vec{u}}}{d \vec{r}}=\operatorname{div} \hat{L} \hat{j}\right) .
$$

As it is discussed above, based on physical arguments, $\hat{j}$ can be uniquely defined, so the deformation velocity field $\dot{\vec{u}}$ can already be determined if $\hat{j}$ is known. Integrating it with respect to time gives the change of the displacement field which is the quantity one can really measure.

\subsection{Problems related to coarse graining}

The dislocation density tensor introduced above is a highly singular quantity. It is infinite along the dislocation lines and vanishes elsewhere. More precisely, it is proportional to a delta function along the dislocation lines. The same holds for the dislocation current density. The conservation law (2.37) guarantees that during the evolution of the dislocation system this delta function does not "spread out", only the shape of the loops can change. This is certainly what we expect physically. This means, however, if we want to follow the evolution of the system we have to follow the track of each dislocation loop as it is done in DDD simulations.

We may hope, like for many other physical systems, to predict the macroscopic response of the dislocation system, we do not need this detailed knowledge of the evolution of the dislocation configuration. One should try to operate with locally averaged quantities. This means, the different quantities, like dislocation density tensor, stress, dislocation current density, etc., are convolved with a window function. In the physics literature the procedure is commonly called as "coarse graining", while in the engineering 
community the term "homogenisation" is more frequently used. One can immediately raise the question what is the appropriate function we should use for the shape of the window function, and what determines its half width. One cannot have a general recipe how to resolve these problems. Nevertheless, we can hope that within certain limits the result obtained by the coarse graining is not sensitive to the actual window function shape and its width. If this is not the case, this clearly indicates that all the microscopical details are important. So, the coarse graining procedure always requires extra care. Beside this, it is important to stress that, before the equations obtained by coarse graining are applied, for predicting the response of the material in a given situation, one always has to study the relevance of the homogenisation.

In order to indicate the difficulties, as a simple example, let us consider a set of parallel edge dislocations with $\pm \vec{b}$ Burgers vectors parallel to the $x$ axis. For this case Eq. (2.33) simplifies to

$$
\nabla^{4} \chi=\frac{2 \mu}{1-\nu}\left(b \frac{\partial}{\partial y}\right) \kappa_{d}
$$

where $\kappa_{d}=\rho_{d+}-\rho_{d-}$ is the signed dislocation density that is a sum of delta functions. If we take the convolution of Eq. (2.44) with a window function $w(\vec{r})$ we obtain that

$$
\int w\left(\vec{r}-\vec{r}^{\prime}\right) \nabla^{\prime 4} \chi\left(\vec{r}^{\prime}\right) d \vec{r}^{\prime}=\frac{2 \mu}{1-\nu} \int w\left(\vec{r}-\vec{r}^{\prime}\right)\left(b \frac{\partial}{\partial y^{\prime}}\right) \kappa_{d}(\vec{r}) d \vec{r}^{\prime} .
$$

After partial integrations we get that

$$
\nabla^{4} \int w\left(\vec{r}-\vec{r}^{\prime}\right) \chi\left(\vec{r}^{\prime}\right) d \vec{r}^{\prime}=\frac{2 \mu}{1-\nu}\left(b \frac{\partial}{\partial y}\right) \int w\left(\vec{r}-\vec{r}^{\prime}\right) \kappa_{d}(\vec{r}) d \vec{r}^{\prime} .
$$

As it can be seen, the coarse grained fields denoted by

$$
<\chi>=\int w\left(\vec{r}-\vec{r}^{\prime}\right) \chi\left(\vec{r}^{\prime}\right) d \vec{r}^{\prime} \quad<\kappa>=\int w\left(\vec{r}-\vec{r}^{\prime}\right) \kappa_{d}\left(\vec{r}^{\prime}\right) d \vec{r}^{\prime}
$$

are related to each other as

$$
\nabla^{4}<\chi>=\frac{\mu}{1-\nu}\left(b \frac{\partial}{\partial y}\right)<\kappa>
$$

that is formally equivalent with Eq. (2.44). With a similar argument, from Eq. (2.31) one can find that

$$
<\sigma>_{11}=-\frac{\partial^{2}<\chi>}{\partial y^{2}}, \quad<\sigma>_{22}=-\frac{\partial^{2}<\chi>}{\partial x^{2}}, \quad<\sigma>_{12}=\frac{\partial^{2}<\chi>}{\partial x \partial y}
$$

This means, the coarse grained fields are related to each other as the "discrete" ones.

However, important information is lost during coarse graining. If we consider two dislocation configurations indicated in Figure 6 and coarse grain them for the same square areas indicated by the boxes, we get the same signed dislocation density value. On the other hand, it is obvious that the response of the two configurations are strongly 

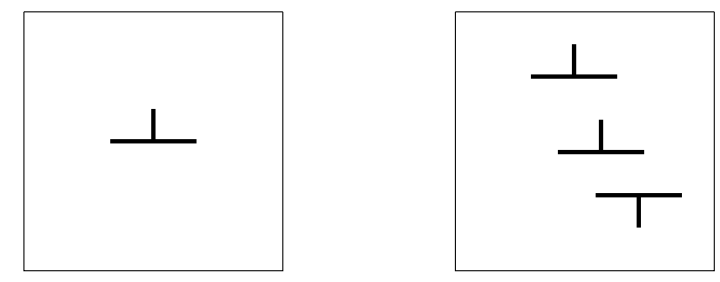

Figure 6. Two strongly different dislocation configurations giving the same $\kappa$ if they are coarse grained for the areas indicated by the boxes.

different, if one applies an external shear. So, in a continuum theory of dislocations, in which we operate with smooth fields, the coarse grained dislocation density tensor is not enough to characterise the state of the system. In the next section we discuss how a continuum theory can be derived from the equation of motion of straight parallel dislocations and what relevant quantities are needed to have an appropriate description of this simple dislocation system on the mesoscopic scale.

\section{Linking micro- to mesoscale for a $2 \mathrm{D}$ dislocation system}

\subsection{Discrete dislocation dynamics simulations in 2D}

In the early 90s due to the fast increase of the available computer power it became possible to investigate the collective properties of dislocations by computer simulations. In DDD simulations the equations of motion of the dislocations (dislocation segments) are integrated numerically. During the past 15 years a vast amount of DDD simulations were performed both in 2D [46, 47, 48, 49, 50, 51, 52, 53, 54, 55, 56] and $3 \mathrm{D}[35,36,37,38$, $39,40,42,43,44,45]$. The detailed descriptions of the numerical methods applied and the results obtained are out of the scope of the paper, but to demonstrate the potentials and the limitations of the numerical simulations we will shortly review simulation results obtained by the author $[50,51]$ on the same system that is studied below in the statistical physics considerations.

In the simulations, the evolution of a system of parallel edge dislocations was studied in a square area with periodic boundary conditions. (This means, the originally 3D problem was simplified to 2D.) The system was subject of unidirectional deformation with a constant external deformation rate $\dot{\epsilon}$. The stress required to keep $\dot{\epsilon}$ constant was calculated. Dislocation motion was allowed in two slip systems (denoted by dotted lines in Figures 7 ) with $60^{0}$ between the two slip directions. The system was oriented for single slip, i.e. in one of the two slip systems the shear stress generated by the external load was much larger than in the other one.

In the simulations reported here only dislocation glide was allowed (i.e. the velocity of the dislocations were parallel to their Burgers vectors). In the equation of motion of the 
dislocations, the inertia term $(m a)$ was neglected beside the friction force $\vec{F}_{f}$ accounting for the energy dissipation during dislocation motion. This is called overdamped dynamics. For simplicity, we assumed that $\vec{F}_{f}$ was proportional to the dislocation velocity, $\vec{F}_{f}=$ $-B \vec{v}$, where the coefficient $B$ is called as dislocation mobility.

Beside the friction force, if the stress is nonzero at the dislocation, the Peach-Koehler force [1]

$$
\vec{F}_{P K}=\vec{l} \times(\hat{\sigma} \vec{b})
$$

also acts on a dislocation, where $\hat{\sigma}$ is the sum of the stress created by the dislocations and the external stress. With these, the equation of motion of the $i$ th dislocation is

$$
\vec{v}_{i}=B^{-1} \vec{s}_{i}\left[\vec{m}_{i}\left(\sum_{j=1}^{N} \hat{\sigma}^{i n d}\left(\vec{r}_{j}-\vec{r}_{i}, \vec{b}_{j}\right)+\hat{\sigma}^{e x t}\right) \vec{b}_{i}\right]
$$

where $\vec{s}_{i}$ and $\vec{m}_{i}$ are unite vectors parallel and perpendicular to the slip direction of the $i$ th dislocation, respectively, $\hat{\sigma}_{\text {ext }}$ is the external stress, and $\hat{\sigma}^{i n d}\left(\vec{r}-\vec{r}_{i}, \vec{b}\right)$ is the stress field created by the $i$ th dislocation (with Burgers vector $\vec{b}$ ). According to Eqs. $(2.31,2.33)$, $\hat{\sigma}^{\text {ind }}$ needs to be determined from the equations

$$
\begin{gathered}
\nabla^{4} \chi^{i n d}=\frac{2 \mu}{1-\nu}\left(b_{1} \frac{\partial}{\partial y}-b_{2} \frac{\partial}{\partial x}\right) \delta\left(\vec{r}-\vec{r}_{i}\right) \\
\sigma_{11}^{i n d}=-\frac{\partial^{2} \chi^{i n d}}{\partial y^{2}}, \quad \sigma_{22}^{i n d}=-\frac{\partial^{2} \chi^{i n d}}{\partial x^{2}}, \quad \sigma_{12}^{i n d}=\frac{\partial^{2} \chi^{i n d}}{\partial x \partial y} .
\end{gathered}
$$

For periodic boundary conditions used in the simulations $\hat{\sigma}^{\text {ind }}$ does not depend on the absolute position of the dislocation position. $\hat{\sigma}^{\text {ind }}$ can be determined numerically either by the Fourier transformation of the above equation or by adding the $\hat{\sigma}_{\infty}^{\text {ind }}$ stress of the appropriate periodic mirror dislocations, where $\hat{\sigma}_{\infty}^{\text {ind }}$ is the stress in an infinite medium. It has to be mentioned here that for other boundary conditions Needleman and coworkers developed an efficient method in which the stress is the sum of the stress the dislocation would create in an infinite medium plus a "compensating" component $\hat{\sigma}^{\text {comp }}$ needed to fulfill the boundary conditions. Since $\hat{\sigma}^{\text {comp }}$ is nonsingular at the dislocations it can be calculated with finite element methods.

During the system evolution dislocation multiplication was allowed with a global and a local conditions. The global condition was set up to reflect the experimental fact that a certain amount of external work is stored in the self-energy of dislocations. To mimic this, if the external work increased a certain amount, a new dislocation dipole was added. Since the new dislocations are generated by the stress as a "local" rule, the new dipole was placed to a random position chosen with a probability proportional to the local stress. For the sake of simplicity dislocation annihilation was not taken into account.

A typical simulation result can be seen in Figure 7. As the stress-time relation obtained indicates, the system has a finite flow stress (the initial part of the curve is linear and reversible). This is the consequence of the relatively narrow random dipole 


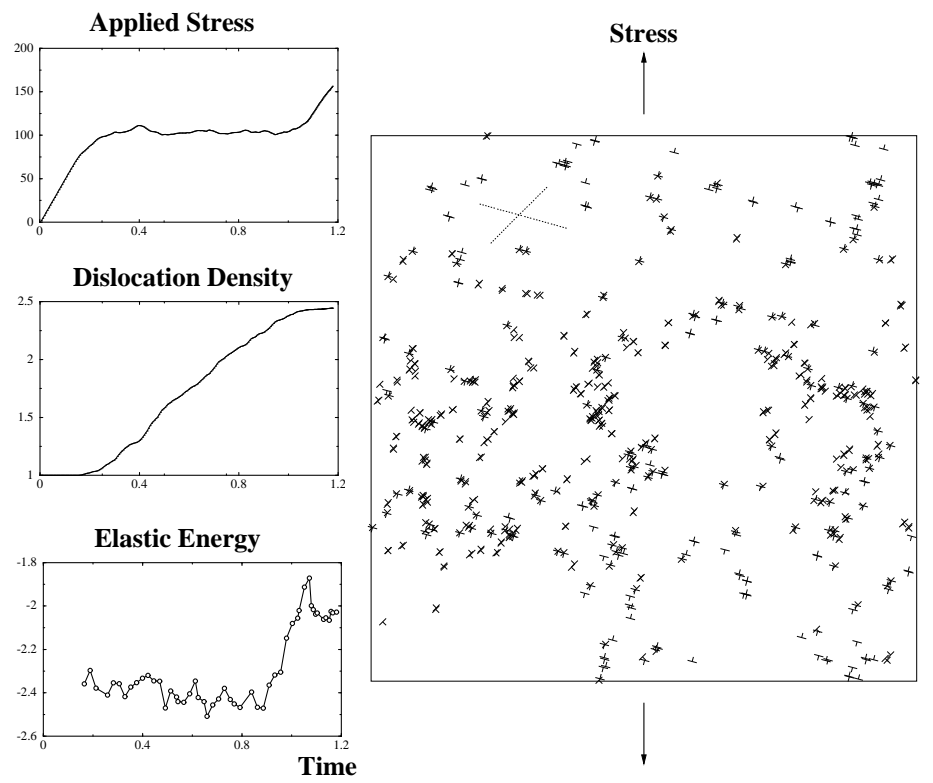

Figure 7. The time variation of stress, dislocation density and elastic energy together with the "final" dislocation configuration obtained on a dislocation system subject of a constant external deformation rate.

initial configuration used. While the external stress is less than the debounding stress of the dipoles there is no "free" dislocation motion, so there is no plastic deformation. The deformation is elastic. After this, the stress-strain curve is practically horizontal with a few dislocations moving in the primary slip system. This stage shows several similarities with the easy glide stage of plastic deformation of single crystals. This stage is followed by a much steeper part of the stress-strain curve. The transition is coupled with a quite sudden rearrangement of the dislocations. A cell like structure starts to form. This stage is similar the to stage II. deformation regime.

We have investigated if the stress increment needed to keep the deformation rate constant results the enhancement of the flow stress. In stage II at a certain stress level the direction of the deformation was reversed and the system was unloaded. After this the system was reloaded again with the same rate applied earlier. As it can be seen in Figure 8 during unloading after a short relaxation period the plastic deformation rate dropped to zero (left bottom curve) and the system continued to deform elastically. During the reloading period macroscopic plastic deformation did not occur until the stress reached the level at which the system was unloaded. This means, that this simple model system shows hardening. Similar results were observed by several other authors $([54,57])$.

The above results demonstrate that already a strongly oversimplified 2D dislocation system is able to reproduce several properties of plastic deformation. Due to the huge computational demand needed for the numerical integration of the equations of motion 


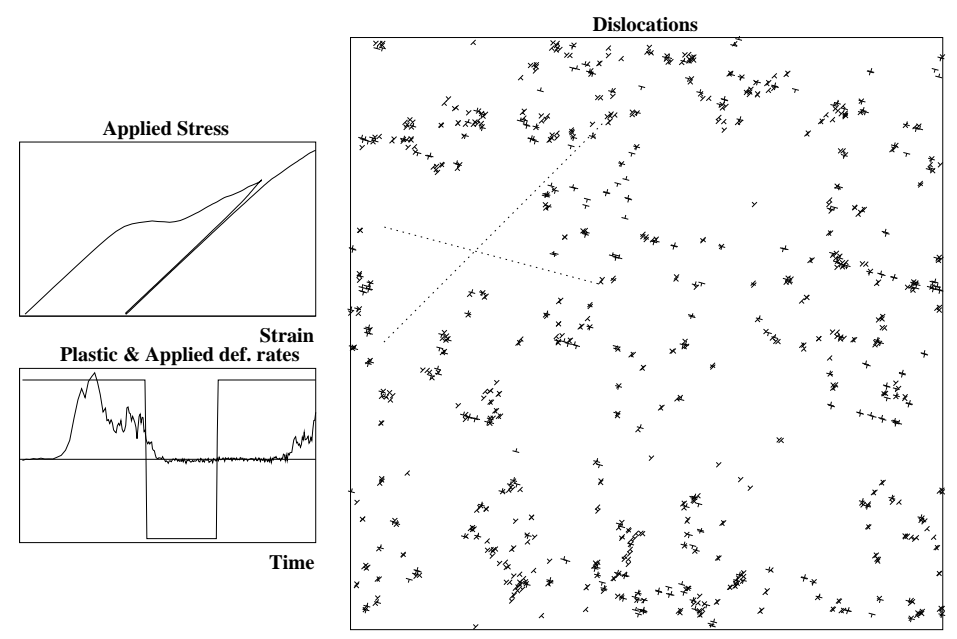

Figure 8. Hardening obtained on the 2D dislocation system.

of the dislocations, one can afford only a couple of thousand dislocations in the simulations. The problem is even more complex in 3D. At the moment about $10^{12} \mathrm{~m}^{-2}$ dislocation density can be reached in a 10x10x10 $\mu \mathrm{m}$ box [45] applying the world largest supercomputers. Although, these numerical investigations are extremely important, at the moment their applicability is very limited in the engineering practice.

\subsection{Continuum theories developed for other systems, analogies and differ- ences}

At the end of the 19th century Boltzmann developed the statistical theory of fluids and gases. The key quantity he introduced is the density function $f(t, \vec{r}, \vec{p})$ giving the probability density of finding an atom at the $(\vec{r}, \vec{p})$ point of the phase space. From the conservation of the phase space volume (Liouville theorem) he obtained that the evolution of $f(t, \vec{r}, \vec{p})$ is described by the relation

$$
\frac{\partial}{\partial t} f(t, \vec{p}, \vec{r})+\frac{\vec{p}}{m} \frac{\partial}{\partial \vec{r}} f(t, \vec{p}, \vec{r})+\vec{F}(\vec{r}) \frac{\partial}{\partial \vec{p}} f(t, \vec{p}, \vec{r})=\frac{\delta f_{c}}{\delta t},
$$

where $m$ is the mass of the atoms, $\vec{F}(\vec{r})$ is the external force and $\delta f_{c} / \delta t$ is the so called collision term accounting for the momentum change occurring at the collision of two atoms. Its actual form is difficult to determine. Since, however, the interaction between atoms is short ranged the collision is a local short event. This means that the collision time $\tau_{c}$ is much shorter than the mean collision free travelling time. As a consequence of this the collision term can be well approximated with a relaxation term leading to the equation

$$
\frac{\partial}{\partial t} f(t, \vec{p}, \vec{r})+\frac{\vec{p}}{m} \frac{\partial}{\partial \vec{r}} f(t, \vec{p}, \vec{r})+\vec{F}(\vec{r}) \frac{\partial}{\partial \vec{p}} f(t, \vec{p}, \vec{r})=-\frac{1}{\tau_{c}}\left(f-f_{\infty}\right),
$$


where $f_{\infty}$ is the equilibrium Boltzmann distribution. An outstanding feature of Eq. (3.6) is that the Navier-Stokes equation of fluid dynamics can be derived from Eq. (3.6).

On the other hand it is obvious that Eq. (3.6) cannot be used for systems where the interaction between the particles is long ranged. Plasma is a typical example for this. The Coulomb interaction between the charged particles is long ranged. The same holds for dislocations. The interaction between straight dislocations is proportional to $1 / r$.

For plasma, Vlasov obtained that the collision term $\delta f_{c} / \delta t$ is the sum of two terms

$$
\frac{\delta f_{c}}{\delta t}=-\vec{F}_{s c}(\vec{r}) \frac{\partial}{\partial \vec{p}} f(t, \vec{p}, \vec{r})+S(\vec{r}),
$$

where $\vec{F}_{s c}(\vec{r})$ is the so called self-consistent field generated by the charge density $\rho_{c}(\vec{r})$

and the electric current density $\vec{j}_{c}(\vec{r})$. The relations between $\rho_{c}(\vec{r}), \vec{j}_{c}(\vec{r})$, and $\vec{F}_{s c}(\vec{r})$ determined by the Maxwell equations, are strongly non-local. However, due to the Debye screening appearing in charged systems, the second term denoted by $S(\vec{r})$ is already local. According to these, for plasma the Boltzmann equation (3.5) reads as

$$
\frac{\partial}{\partial t} f(t, \vec{p}, \vec{r})+\frac{\vec{p}}{m} \frac{\partial}{\partial \vec{r}} f(t, \vec{p}, \vec{r})+\left[\vec{F}(\vec{r})+\vec{F}_{s c}(\vec{r})\right] \frac{\partial}{\partial \vec{p}} f(t, \vec{p}, \vec{r})=S(\vec{r}) .
$$

As it is explained below, a similar equation can be derived for straight dislocations. Certainly, the special properties of the dislocation-dislocation interaction and the dissipative nature of dislocation motion have to be taken into account.

\subsection{Hierarchy of the different order density functions}

Let us consider $N$ straight parallel edge dislocations. As a first step let us assume that each dislocation has the same Burgers vector $\vec{b}$ parallel to the $x$ axis. This simplification is needed only to have shorter equations. The results obtained can be easily generalised. In order to get results that are physically relevant some generalisation is definitely needed.

For this case the equation of motion of the dislocations given by the general form (3.2) simplifies to

$$
\vec{v}_{i}=B^{-1} \vec{b}\left(\sum_{j \neq i}^{N} \tau_{i n d}\left(\vec{r}_{i}-\vec{r}_{j}\right)+\tau_{e x t}\right),
$$

where $\tau_{\text {ext }}$ is the external shear and $\tau_{\text {ind }}(\vec{r})$ is the shear stress created by a dislocation. In an infinite isotropic medium

$$
\tau_{\text {ind }}(\vec{r})=\frac{b \mu}{2 \pi(1-\nu)} \frac{x\left(x^{2}-y^{2}\right)}{\left(x^{2}+y^{2}\right)^{2}} .
$$

As it is well known from statistical physics, instead of giving the time dependence of the coordinates of the $N$ particles one can describe the state and the evolution of the system with the $N$ particle probability density function $f_{N}$ varying in the $6 \mathrm{~N}$ dimensional phase space. Although, the dislocations form a nonconservative system, some of the results of statistical mechanics can be applied. Since the equations of motion of dislocations 
are only first order differential equations (assuming overdamped motion) for the problem considered, $f_{N}$ is a $2 \mathrm{~N}$ dimensional function of the dislocation coordinates. By definition $f_{N}\left(t, \vec{r}_{1}, \vec{r}_{2} \ldots \vec{r}_{N}\right) d^{2} \vec{r}_{1} d^{2} \vec{r}_{2}, . . d^{2} \vec{r}_{N}$ is the probability of finding the $N$ dislocations in the $d^{2} \vec{r}_{1} d^{2} \vec{r}_{2} . . d^{2} \vec{r}_{N}$ vicinity of the $\vec{r}_{1}, \vec{r}_{2} \ldots \vec{r}_{N}$ points at time $t$.

If we assume that the number of dislocations is conserved (later this restriction will be lifted), $f_{N}$ has to fulfill the conservation law [58]

$$
\begin{aligned}
f_{N}( & \left.t, \vec{r}_{1}, \vec{r}_{2}, \ldots, \vec{r}_{N}\right) d^{2} \vec{r}_{1} d^{2} \vec{r}_{2}, \ldots, d^{2} \vec{r}_{N}= \\
& f_{N}\left(t+\Delta t, \vec{r}_{1}+\vec{v}_{1} \Delta t, \vec{r}_{2}+\vec{v}_{2} \Delta t \ldots \vec{r}_{N}+\vec{v}_{N} \Delta t\right) \\
& \times d^{2}\left(\vec{r}_{1}+\vec{v}_{1} \Delta t\right) d^{2}\left(\vec{r}_{2}+\vec{v}_{2} \Delta t\right) \ldots d^{2}\left(\vec{r}_{N}+\vec{v}_{N} \Delta t\right) .
\end{aligned}
$$

The above relation reflects the simple fact that the probability of finding a dislocation at a certain point can change only if the dislocation moves from one point to another one. It is interesting to mention that in contrast with the conservative systems

$$
\begin{aligned}
& f_{N}\left(t, \vec{r}_{1}, \vec{r}_{2}, \ldots, \vec{r}_{N}\right) \neq \\
& \quad f_{N}\left(t+\Delta t, \vec{r}_{1}+\vec{v}_{1} \Delta t, \vec{r}_{2}+\vec{v}_{2} \Delta t, \ldots, \vec{r}_{N}+\vec{v}_{N} \Delta t\right),
\end{aligned}
$$

This is the consequence of that the $d \vec{r}_{1} d \vec{r}_{2} \ldots d \vec{r}_{N}$ volume is not conserved during the evolution of the system.

After some simple algebraic manipulations Eq. (3.11) can be rewritten into a partial differentiation equation

$$
\frac{\partial f_{N}}{\partial t}+\sum_{i=1}^{N} \frac{\partial}{\partial \vec{r}_{i}}\left\{f_{N}\left(t, \vec{r}_{1}, \vec{r}_{2}, \ldots, \vec{r}_{N}\right) \vec{v}_{i}\right\}=0 .
$$

By substituting the left hand side of Eq. (3.9) into $\vec{v}_{i}$ we get that

$$
\frac{\partial f_{N}}{\partial t}+\sum_{i \neq j}^{N} \frac{\partial}{\partial \vec{r}_{i}}\left\{f_{N} \vec{F}\left(\vec{r}_{i}-\vec{r}_{j}\right)\right\}=0
$$

where $\vec{F}(\vec{r})=\vec{b} \tau_{\text {ind }}(\vec{r})$. (B is dropped out from Eq. (3.14). With the appropriate selection of the time unit one can always take $B=1$.) For the sake of simplicity in the above equation the external shear was not taken into account. It is important to note that Eq. (3.14) is mathematically equivalent with the original equations of motion of the dislocations (3.9). To find a solution of the two equations are equally difficult.

For many applications, however, we do not need that detailed description represented by the $N$ particle probability density function. A less detailed description of the system is the $k$-th order probability density function defined as

$$
f_{k}\left(\vec{r}_{1}, \vec{r}_{2}, . ., \vec{r}_{k}\right)=\iint . . \int f_{N}\left(t, \vec{r}_{1}, \vec{r}_{2} \ldots \vec{r}_{N}\right) d^{2} \vec{r}_{k+1} d^{2} \vec{r}_{k+2} \ldots d^{2} \vec{r}_{N} .
$$

After integrating Eq. (3.14) with respect to the variables $\vec{r}_{k+1}, \vec{r}_{k+2}, . . \vec{r}_{N}$, from the above definition of $f_{k}(3.15)$ we obtain that

$$
\frac{\partial f_{k}}{\partial t}=-\sum_{i=1}^{N} \sum_{j=1, j \neq i}^{N} \quad \int \frac{\partial}{\partial \vec{r}_{i}}\left\{f_{N} \vec{F}\left(\vec{r}_{i}-\vec{r}_{j}\right)\right\} d^{2} \vec{r}_{k+1} d^{2} \vec{r}_{k+2} \ldots d^{2} \vec{r}_{N} .
$$


The double sum at the right hand side of the equation can be split into three parts

$$
\begin{aligned}
& \sum_{i=1}^{N} \sum_{j=1, j \neq i}^{N} \int \frac{\partial}{\partial \vec{r}_{i}}\left\{f_{N} \vec{F}\left(\vec{r}_{i}-\vec{r}_{j}\right)\right\} d^{2} \vec{r}_{k+1} d^{2} \vec{r}_{k+2} \ldots d^{2} \vec{r}_{N}= \\
& \quad \sum_{i=1}^{k} \sum_{j=1, j \neq i}^{k} \frac{\partial}{\partial \vec{r}_{i}}\left\{f_{k} \vec{F}\left(\vec{r}_{i}-\vec{r}_{j}\right)\right\} \\
& \quad+\sum_{i=1}^{k} \sum_{j=k+1}^{N} \int \frac{\partial}{\partial \vec{r}_{i}}\left\{f_{N} \vec{F}\left(\vec{r}_{i}-\vec{r}_{j}\right)\right\} d^{2} \vec{r}_{k+1} d^{2} \vec{r}_{k+2} \ldots d^{2} \vec{r}_{N} \\
& \quad+\sum_{i=k+1}^{N} \sum_{j=1, j \neq i}^{N} \int \frac{\partial}{\partial \vec{r}_{i}}\left\{f_{N} \vec{F}\left(\vec{r}_{i}-\vec{r}_{j}\right)\right\} d^{2} \vec{r}_{k+1} d^{2} \vec{r}_{k+2} \ldots d^{2} \vec{r}_{N}
\end{aligned}
$$

The last term is the integral of a div, so it can be transformed into a contour integral along the border of the system. Assuming that the distribution functions tend to zero fast enough at infinity, this term vanishes. Taking into account that $f_{N}$ needs to be invariant with respects to swapping the coordinates of two dislocations we get that

$$
\begin{aligned}
\frac{\partial f_{k}}{\partial t} & +\sum_{i=1}^{k} \sum_{j=1, j \neq i}^{k} \frac{\partial}{\partial \vec{r}_{i}}\left\{f_{k} \vec{F}\left(\vec{r}_{i}-\vec{r}_{j}\right)\right\} \\
& +(N-k) \int \frac{\partial}{\partial \vec{r}_{i}}\left\{f_{k+1} \vec{F}\left(\vec{r}_{i}-\vec{r}_{k+1}\right)\right\} d^{2} \vec{r}_{k+1}=0 .
\end{aligned}
$$

As it can be seen the equation for the $k$-th order probability distribution function depends on the $k+1$-th order one. So, the reduction procedure applied results a hierarchy of the equations. In fluid dynamics and plasma physics this is called as BBGKY hierarchy.

For our further consideration the equations for $f_{1}$ and $f_{2}$ play an important role, so we give their explicit forms [58]:

$$
\frac{\partial \rho_{1}\left(\vec{r}_{1}, t\right)}{\partial t}+\int \frac{\partial}{\partial \vec{r}_{1}}\left\{\rho_{2}\left(\vec{r}_{1}, \vec{r}_{2}, t\right) \vec{F}\left(\vec{r}_{1}-\vec{r}_{2}\right)\right\} d^{2} \vec{r}_{2}=0
$$

and

$$
\begin{aligned}
& \frac{\partial \rho_{2}\left(\vec{r}_{1}, \vec{r}_{2}, t\right)}{\partial t}+\left(\frac{\partial}{\partial \vec{r}_{1}}-\frac{\partial}{\partial \vec{r}_{2}}\right) \rho_{2}\left(\vec{r}_{1}, \vec{r}_{2}, t\right) \vec{F}\left(\vec{r}_{1}-\vec{r}_{2}\right) \\
& \quad+\frac{\partial}{\partial \vec{r}_{1}} \int \rho_{3}\left(\vec{r}_{1}, \vec{r}_{2}, \vec{r}_{3}, t\right) \vec{F}\left(\vec{r}_{1}-\vec{r}_{3}\right) d^{2} \vec{r}_{3}+1 \leftrightarrow 2=0
\end{aligned}
$$

where the notations $\rho_{1}=N f_{1}, \rho_{2}=N(N-1) f_{2}, \rho_{3}=N(N-1)(N-2) f_{3}$ were introduced. The advantage of using these quantities is that, in contrast with the probability densities $f_{1}, f_{2}$ and $f_{3}$ normalised to 1 , they are system size independent. They are commonly referred to as one, two and three particle density functions, respectively.

It is useful to show that Eq. (3.19) can be derived with another method, too [59]. This can help to have a deeper understanding of the physical meaning of the equation 
obtained. As a first step let us multiply (3.9) with $\delta\left(\vec{r}-\vec{r}_{i}\right)$ and take its derivative with respect to $\vec{r}$ :

$$
\frac{d}{d \vec{r}}\left\{\frac{d \vec{r}_{i}}{d t} \delta\left(\vec{r}-\vec{r}_{i}\right)\right\}=\frac{d}{d \vec{r}}\left\{\left(\sum_{j \neq i}^{N} \vec{F}\left(\vec{r}_{i}-\vec{r}_{j}\right)\right) \delta\left(\vec{r}-\vec{r}_{i}\right)\right\} .
$$

It is useful to introduce the "discrete" dislocation density

$$
\rho_{d}(\vec{r})=\sum_{i=1}^{N} \delta\left(\vec{r}-\vec{r}_{i}\right)
$$

that is the same as $\rho_{d+}$ defined in subsection 2.4, but since in the present analysis only one type of dislocation was considered, the subscript + was dropped. With this, the left hand side of Eq. (3.21) can be rewritten into a weighted integral. Furthermore, taking into account that

$$
\frac{d}{d \vec{r}}\left\{\frac{d \vec{r}_{i}}{d t} \delta\left(\vec{r}-\vec{r}_{i}\right)\right\}=-\frac{d \vec{r}_{i}}{d t} \frac{d}{d \vec{r}_{i}} \delta\left(\vec{r}-\vec{r}_{i}\right)=-\frac{d}{d t} \delta\left(\vec{r}-\vec{r}_{i}\right),
$$

from Eq. (3.21) we get that

$$
\begin{aligned}
- & \frac{d}{d t} \delta\left(\vec{r}-\vec{r}_{i}\right) \\
& =\frac{d}{d \vec{r}}\left\{\left(\int \vec{F}\left(\vec{r}-\vec{r}^{\prime}\right)\left[\rho_{d}\left(\vec{r}^{\prime}\right)-\delta\left(\vec{r}-\vec{r}^{\prime}\right)\right] d^{2} \vec{r}^{\prime}\right) \delta\left(\vec{r}-\vec{r}_{i}\right)\right\}
\end{aligned}
$$

(where $\delta\left(\vec{r}-\vec{r}^{\prime}\right)$ beside $\rho_{d}\left(\vec{r}^{\prime}\right)$ is needed to avoid self dislocation interaction.) By summing up with respect to $i$ we conclude

$$
-\frac{d}{d t} \rho_{d}(\vec{r})=\frac{d}{d \vec{r}}\left\{\left(\int \vec{F}\left(\vec{r}-\vec{r}^{\prime}\right)\left[\rho_{d}\left(\vec{r}^{\prime}\right)-\delta\left(\vec{r}-\vec{r}^{\prime}\right)\right] d^{2} \vec{r}^{\prime}\right) \rho_{d}(\vec{r})\right\},
$$

which is a nonlinear strongly non-local equation for the "discrete" dislocation density $\rho_{d}(\vec{r})$. Like it was done with the field equation (2.44), to get rid of the singular character of $\rho_{d}(\vec{r})$ we can coarse grain Eq. (3.25). By introducing the coarse grained quantities

$$
\begin{gathered}
\rho_{1}(\vec{r})=<\rho_{d i s c}(\vec{r})> \\
\rho_{2}\left(\vec{r}_{1}, \vec{r}_{2}\right)=<\rho_{d i s c}\left(\vec{r}_{1}\right) \rho_{d i s c}\left(\vec{r}_{2}\right)-\rho_{d i s c}\left(\vec{r}_{1}\right) \delta\left(\vec{r}_{1}-\vec{r}_{2}>,\right.
\end{gathered}
$$

we get back Eq. (3.19) derived earlier. The procedure applied above clearly shows that the form of Eq. (3.19) does not depend on the actual form of the window function applied for the coarse graining. However, $\rho_{1}(\vec{r})$ and $\rho_{2}\left(\vec{r}_{1}, \vec{r}_{2}\right)$ can depend on $w(\vec{r})$ chosen. Certainly, this is not a problem until we do not assume some relation between $\rho_{1}(\vec{r})$ and $\rho_{2}\left(\vec{r}_{1}, \vec{r}_{2}\right)$. We can say that Eq. (3.19) is exact but it is not enough to describe the time evolution of the dislocation density. 
Before we discuss how a closed theory can be obtained, the above results have to be generalised for the case where Burgers vector of the dislocations are not the same. The simplest generalisation is if we allow that the Burgers vectors of the dislocations can differ in sign. This is still a strong simplification of a real dislocation ensemble but an important step forward. Without going into the details with a similar procedure explained above one can find that

$$
\begin{aligned}
& \frac{\partial \rho_{+}\left(\vec{r}_{1}, t\right)}{\partial t} \\
& \quad+\vec{b} \frac{\partial}{\partial \vec{r}_{1}}\left[\rho_{+}\left(\vec{r}_{1}, t\right) \tau_{\text {ext }}+\int\left\{\rho_{++}\left(\vec{r}_{1}, \vec{r}_{2}, t\right)-\rho_{+-}\left(\vec{r}_{1}, \vec{r}_{2}, t\right)\right\} \tau_{\text {ind }}\left(\vec{r}_{1}-\vec{r}_{2}\right) d \vec{r}_{2}\right]=0 \\
& \frac{\partial \rho_{-}\left(\vec{r}_{1}, t\right)}{\partial t} \\
& +\vec{b} \frac{\partial}{\partial \vec{r}_{1}}\left[-\rho_{-}\left(\vec{r}_{1}, t\right) \tau_{\text {ext }}+\int\left\{\rho_{--}\left(\vec{r}_{1}, \vec{r}_{2}, t\right)-\rho_{-+}\left(\vec{r}_{1}, \vec{r}_{2}, t\right)\right\} \tau_{\text {ind }}\left(\vec{r}_{1}-\vec{r}_{2}\right) d \vec{r}_{2}\right]=0
\end{aligned}
$$

where $\vec{b}$ is the Burgers vector of the positive signed dislocations. The subscripts "+" and "-" indicate the sign of the Burgers vector the different density functions are corresponding to. We mention here that the negative signs in front of $\rho_{+-}$and $\rho_{-+}$in Eqs. (3.28) and (3.29) come from the simple fact that the interaction force acting between dislocations with opposite signs is $-F_{\text {ind }}$.

By adding and substituting the two equations we obtain:

$$
\begin{aligned}
& \frac{\partial \rho\left(\vec{r}_{1}, t\right)}{\partial t}+\vec{b} \frac{\partial}{\partial \vec{r}_{1}}\left[\kappa\left(\overrightarrow{r_{1}}, t\right) \tau_{\text {ext }}+\int\left\{\rho_{++}\left(\vec{r}_{1}, \vec{r}_{2}, t\right)+\rho_{--}\left(\vec{r}_{1}, \vec{r}_{2}, t\right)\right.\right. \\
& \left.\left.-\rho_{+-}\left(\vec{r}_{1}, \vec{r}_{2}, t\right)-\rho_{-+}\left(\vec{r}_{1}, \vec{r}_{2}, t\right)\right\} \tau_{\text {ind }}\left(\vec{r}_{1}-\vec{r}_{2}\right) d \vec{r}_{2}\right]=0, \\
& \frac{\partial \kappa\left(\vec{r}_{1}, t\right)}{\partial t}+\vec{b} \frac{\partial}{\partial \vec{r}_{1}}\left[\rho\left(\overrightarrow{r_{1}}, t\right) \tau_{\text {ext }}+\int\left\{\rho_{++}\left(\vec{r}_{1}, \vec{r}_{2}, t\right)-\rho_{--}\left(\vec{r}_{1}, \vec{r}_{2}, t\right)\right.\right. \\
& \left.\left.-\rho_{+-}\left(\vec{r}_{1}, \vec{r}_{2}, t\right)+\rho_{-+}\left(\vec{r}_{1}, \vec{r}_{2}, t\right)\right\} \tau_{\text {ind }}\left(\vec{r}_{1}-\vec{r}_{2}\right) d \vec{r}_{2}\right]=0
\end{aligned}
$$

where $\rho(\vec{r}, t)=\rho_{+}(\vec{r}, t)+\rho_{-}(\vec{r}, t)$ is the total and $\kappa(\vec{r}, t)=\rho_{+}(\vec{r}, t)-\rho_{-}(\vec{r}, t)$ is the signed dislocation density. ( $\kappa$ is the same as $\langle\kappa\rangle$ introduced in Eq. (2.47) but to have shorter equations the brackets $\langle. .>$ were omitted .)

\subsection{Evolution of the plastic shear}

Before we discuss how a closed theory can be obtained for the evolution of $\rho$ and $\kappa$ it is useful to analyse the evolution of plastic shear. For the dislocation geometry considered the only non-vanishing component of the dislocation density tensor is

$$
\alpha_{31}=b \kappa .
$$

According to the definition of $\hat{\alpha}$ given by Eq. (2.8) for the plane problem considered the only component of the plastic distortion contributing to $\alpha_{31}$ is $\beta_{21}^{p}$ and

$$
b \kappa=-\frac{\partial \beta_{21}^{p}}{\partial x} \text {. }
$$


With the notation $\gamma=\beta_{21}^{p}$ commonly used, the above equation can be rewritten as

$$
\kappa=-\frac{\vec{b}}{b^{2}} \frac{d \gamma}{d \vec{r}}
$$

i.e. $\kappa$ is proportional to the gradient of the plastic shear. With other words, this means, to get spatially varying plastic shear one has to introduce dislocations. This is why $\kappa$ is often called geometrically necessary dislocation (GND) density.

Taking the time derivative of Eq. (3.34) we get that

$$
\frac{\partial \kappa}{\partial t}=-\frac{\vec{b}}{b^{2}} \frac{d \dot{\gamma}}{d \vec{r}} .
$$

By comparing this with Eq. (3.31) we obtain an explicit expression for the plastic shear rate $\dot{\gamma}$ :

$$
\begin{aligned}
\dot{\gamma}= & b^{2}\left[\rho\left(\overrightarrow{r_{1}}, t\right) \tau_{e x t}\right. \\
& \left.+\int\left\{\rho_{++}\left(\vec{r}_{1}, \vec{r}_{2}, t\right)-\rho_{--}\left(\vec{r}_{1}, \vec{r}_{2}, t\right)-\rho_{+-}\left(\vec{r}_{1}, \vec{r}_{2}, t\right)+\rho_{-+}\left(\vec{r}_{1}, \vec{r}_{2}, t\right)\right\} \tau_{\text {ind }}\left(\vec{r}_{1}-\vec{r}_{2}\right) d \vec{r}_{2}\right] .
\end{aligned}
$$

\subsection{Self-consistent field approximation}

In order to have a closed continuum theory describing the evolution of the dislocation system, the (3.18) hierarchy of equations has to be cut at some order. In order to do this, from some considerations independent from the Eq. (3.18) we have to give how the density functions with order higher than a given one can be built from the lower order ones. The simplest possible assumption is that the two particle density functions are the products of the one particle density functions [59], i.e.

$$
\rho_{s s^{\prime}}\left(\vec{r}_{1}, \vec{r}_{2}, t\right)=\rho_{s}\left(\vec{r}_{1}\right) \rho_{s^{\prime}}\left(\vec{r}_{2}\right), s, s^{\prime} \in\{+,-\} .
$$

This means, that the short range correlations are neglected. As it is explained below this leads to a self-consistent field theory. Similar approximation is often used in plasma physics.

By substituting Eq. (3.37) into Eqs. $(3.30,3.31)$ we arrive at

$$
\begin{aligned}
& \frac{\partial \rho(\vec{r}, t)}{\partial t}+\vec{b} \frac{\partial}{\partial \vec{r}}\left[\kappa(\vec{r}, t)\left\{\tau_{s c}(\vec{r}, t)+\tau_{e x t}\right\}\right]=0, \\
& \frac{\partial \kappa(\vec{r}, t)}{\partial t}+\vec{b} \frac{\partial}{\partial \vec{r}}\left[\rho(\vec{r}, t)\left\{\tau_{s c}(\vec{r}, t)+\tau_{e x t}\right\}\right]=0,
\end{aligned}
$$

where

$$
\tau_{s c}(\vec{r})=\int \kappa\left(\vec{r}_{1}, t\right) \tau_{i n d}\left(\vec{r}-\vec{r}_{1}\right) d \vec{r}_{1}
$$


is a field (with stress dimension) created by the coarse grained signed dislocation density. $\tau_{s c}$ is often called as self-consistent stress field. However, $\tau_{s c}$ is not a "new" quantity. From Eq. (3.10) one can see that $\tau_{s c}$ fulfill the field equations

$$
\Delta^{2} \chi=\frac{2 b \mu}{(1-\nu)} \frac{\partial}{\partial y} \kappa(\vec{r}), \quad \tau_{s c}=\frac{\partial^{2}}{\partial x \partial y} \chi
$$

If we compare Eq. $(3.41)$ with Eqs. $(2.48,2.49)$ we can see that $\tau_{s c}$ is nothing but the coarse grained shear stress $\langle\sigma\rangle_{12}$.

It is important to note that dislocation multiplication and annihilation can also be taken into account by adding an $f\left(\rho, \tau_{\text {ext }}+\tau_{s c}, \ldots\right)$ source term to the right hand side of Eq. (3.38):

$$
\frac{\partial \rho(\vec{r}, t)}{\partial t}+\vec{b} \frac{\partial}{\partial \vec{r}}\left[\kappa(\vec{r}, t)\left\{\tau_{s c}(\vec{r}, t)+\tau_{\text {ext }}\right\}\right]=f\left(\rho, \tau_{\text {ext }}+\tau_{s c}, \ldots\right) .
$$

Determining the actual form of the source term is a difficult issue. We will come back to this problem later on, but it has to be stressed at this point that Eq. (3.39) has to remain unchanged because it expresses that the total net Burgers vector of the dislocation system cannot change during deformation.

\subsection{Stability analysis}

Since dislocation multiplication is a "local" event, it is plausible to assume that in a given point the source term $f\left(\rho, \tau_{e x t}+\tau_{s c}, \ldots\right)$ depends on only the local values of the dislocation density and the total shear stress $\tau=\tau_{e x t}+\tau_{s c}$ [59]. It is easy to see that in this case Eqs. $(3.39,3.42)$ have a trivial solution that is $\kappa(\vec{r}, t)=0$, and $\rho(\vec{r}, t)=\rho_{0}(t)$ where $\rho_{0}(t)$ is the solution of equation

$$
\frac{d \rho_{0}}{d t}=f\left(\rho_{0}, \tau_{e x t}\right)
$$

Since, however, the equations are strongly nonlinear it is important to analyse the stability of this homogeneous solution [59]. For this, let us linearise Eqs. $(3.38,3.39,3.41)$ around the trivial solution

$$
\begin{gathered}
\frac{d}{d t} \rho^{\prime}+\frac{\partial}{\partial x}\left\{b \tau_{e x t} \kappa^{\prime}\right\}=\left.\frac{\partial f}{\partial \rho}\right|_{\rho=\rho_{0}} \rho^{\prime}+\left.\frac{\partial f}{\partial \tau}\right|_{\tau=\tau_{e x t}} \tau^{\prime}, \\
\frac{d}{d t} \kappa^{\prime}+\frac{\partial}{\partial x}\left\{b\left(\tau_{e x t} \rho^{\prime}+\rho_{0} \tau^{\prime}\right)\right\}=0 \\
\Delta^{2} \chi^{\prime}=\frac{2 b \mu}{(1-\nu)} \frac{\partial}{\partial y} \kappa^{\prime}, \quad \tau^{\prime}=\frac{\partial^{2}}{\partial x \partial y} \chi^{\prime},
\end{gathered}
$$

where $\kappa^{\prime}, \rho^{\prime}=\rho-\rho_{0}$, and $\tau^{\prime}$ are small perturbations. The solution of the linearised equations can be found in the form (assuming that $\rho_{0}(t)$ varies slowly in time)

$$
\left[\begin{array}{c}
\tau^{\prime}(\vec{r}, t) \\
\kappa^{\prime}(\vec{r}, t) \\
\rho^{\prime}(\vec{r}, t) \\
\chi^{\prime}(\vec{r}, t)
\end{array}\right]=\left[\begin{array}{c}
\overline{\tau^{\prime}} \\
\frac{\bar{\kappa}}{\overline{\rho^{\prime}}} \\
\overline{\chi^{\prime}}
\end{array}\right] \exp \left\{\lambda t+i\left(q_{x} x+q_{y} y\right)\right\}
$$


Substituting this form into the Eqs. (3.44-3.45) one can find that $\lambda$ and the wave vector $\left(q_{x}, q_{y}\right)$ have to fulfill the characteristic equation

$$
\left|\begin{array}{cc}
\lambda-\left.\frac{\partial f}{\partial \rho}\right|_{\rho=\rho_{0}} & i q_{x} b \tau_{\text {ext }}+\left.i \frac{T(\Phi)}{q_{x}} \frac{\partial f}{\partial \tau}\right|_{\tau=\tau_{\text {ext }}} \\
i q_{x} b \tau_{\text {ext }} & \lambda+\rho_{0} T(\Phi)
\end{array}\right|=0,
$$

where

$$
T(\Phi)=\frac{2 b^{2} \mu}{(1-\nu)} \frac{q_{x}^{2} q_{y}^{2}}{\left(q_{x}^{2}+q_{y}^{2}\right)^{2}}=\frac{b^{2} \mu}{2(1-\nu)} \sin ^{2}(2 \Phi),
$$

in which $\Phi$ is the angle between the $x$ axis and the wave vector.

The homogeneous solution is stable only if the real part of $\lambda$ is non-positive for any wave vector $\left(q_{x}, q_{y}\right)$. This guarantees that there is no growing perturbation.

As a first step let us analyse the stability of the homogeneous solution if the total number of dislocations is conserved, i.e. if the source term $f(\rho, \tau)$ is zero. In this case the solution of the characteristic equation (3.48) reads as

$$
\lambda_{1,2}=\frac{-T(\Phi) \rho_{0} \pm \sqrt{T(\Phi)^{2} \rho_{0}^{2}-4\left(b \tau_{e x t}\right)^{2} q_{x}^{2}}}{2} .
$$

Since, $T(\Phi)$ is non-negative the real part of both $\lambda_{1}$ and $\lambda_{2}$ are non-positive, so in the absence of source term the homogeneous solution is stable. However, an important feature of the linearised equations is that if the wave vector is parallel either to the $x$ or the $y$ axes the real parts of $\lambda_{1}$ and $\lambda_{2}$ vanish. This means, periodic perturbations which are either parallel or perpendicular to the Burgers vector neither growth nor die out, they are marginally stable.

To see the influence of the source term $f(\rho, \tau)$ it is enough to study the sum of the two roots $\lambda_{1}$ and $\lambda_{2}$. From Eq. (3.48) we get that

$$
\lambda_{1}+\lambda_{2}=-T(\Phi) \rho_{0}+\left.\frac{\partial f}{\partial \rho}\right|_{\rho=\rho_{0}} .
$$

Since $T(\Phi)$ vanishes for $\Phi=0$ and $\Phi=90^{\circ}$, if $\partial f / \partial \rho$ is positive, there is a wave vector domain where at least one of the two $\lambda$-s is positive. This means, if $\partial f / \partial \rho>0$ the homogeneous solution is not stable any more.

\subsection{Numerical studies}

Since the self-consistent field equations $(3.39,3.42)$ are complicated nonlinear equations, studying the properties of their solutions requires numerical investigations. The numerical results presented in this paper [59] were obtained on a $128 \times 128$ grid defined in a square simulation area with periodic boundary conditions. The time integration of Eqs. $(3.39,3.42)$ was carried out by the Newton method. The internal stress was determined from Eq. (3.41) by fast Fourier transformation. In each calculation a constant dislocation density and a random $\kappa$ distribution was used as initial configuration. For the source term the form

$$
f(\rho, \tau)=C\left(\dot{\gamma} \tau-0.09 b^{2} \mu^{2} \rho^{2}\right)=C\left(\rho \tau^{2}-0.09 b^{2} \mu^{2} \rho^{2}\right)
$$


was used. In expression (3.52) the first, dislocation creation term mimics the experimental observation that a certain amount of plastic work is stored as the self energy of dislocations. The second, annihilation term simply expresses that annihilation requires to have two dislocations close to each other. The constants are determined according to the Taylor relation

$$
\tau_{\text {ext }}=0.3 b \mu \sqrt{\rho_{0}}
$$

which we expect to hold at steady state.

Since, apart from $C$, the size of the simulation area and the material parameters can be scaled out from the equations the input parameters $\left(\rho(t=0, \vec{r}), \kappa(t=0, \vec{r}), \tau_{\text {ext }}, C\right)$ and the results of the numerical calculations are given in arbitrary units.
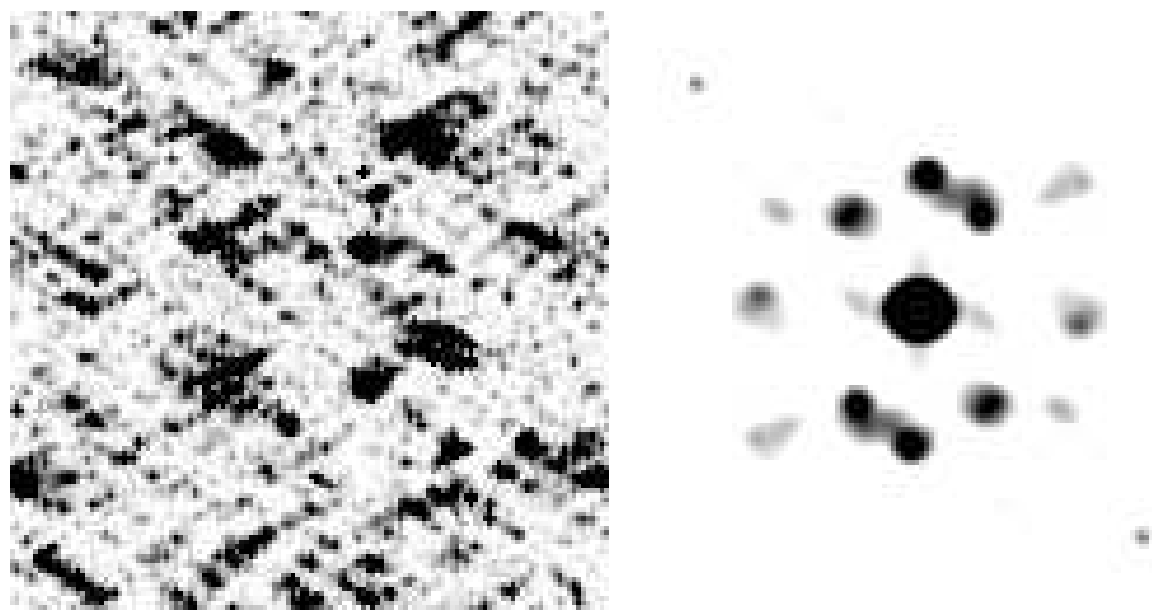

Figure 9. Dislocation density map (left box) and its autocorrelation function $A(\vec{r})=$ $\int \rho\left(\vec{r}-\vec{r}^{\prime}\right) \rho\left(\vec{r}^{\prime}\right) d \vec{r}^{\prime}$ (right box) obtained at periodic external load. (The glide direction is horizontal.)

A typical snapshot obtained at periodic external stress can be seen in Figure 9. Both the dislocation density map (left box) and its autocorrelation function (right box) indicate that the dislocations tend to form a more or less periodic arrangement of dense regions. The dislocation pattern developed is very similar to the so called "matrix" structure experimentally observed on fatigued fcc single crystals [60] (see Figure 10).

It should be mentioned, that if the gradients in the dislocation pattern developing during the deformation become large the numerical solution blows up indicating that the self-consistent field theory can become unstable. In order to resolve this problem the influence of dislocation-dislocation correlation has to be analysed.

\subsection{The role of dislocation-dislocation correlation}

The self-consistent field theory explained above was obtained by assuming that the two particle density functions are the product of the corresponding one particle densities. 


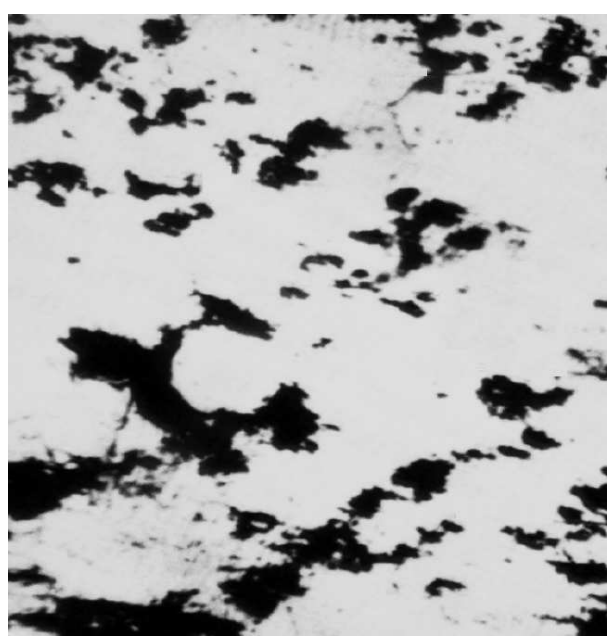

Figure 10. Dislocation "matrix" structure obtained on fatigued $\mathrm{Cu}$ single crystal oriented for single slip [60].

Without restricting generality, the two particle density functions can be given in the form:

$$
\rho_{s s^{\prime}}\left(\vec{r}_{1}, \vec{r}_{2}, t\right)=\rho_{s}\left(\vec{r}_{1}\right) \rho_{s^{\prime}}\left(\vec{r}_{2}\right)\left(1+d_{s s^{\prime}}\left(\vec{r}_{1}, \vec{r}_{2}\right)\right) \quad s, s^{\prime} \in\{+,-\}
$$

where $d_{s s^{\prime}}$ is called dislocation-dislocation correlation function. In order to be able to say something about the correlation function as a first step it is useful to analyse the properties of dislocation-dislocation correlations in an originally homogeneous relaxed dislocation system [61, 62]. Although the BBGKY hierarchy explained earlier gives the possibility to investigate the properties of $d_{s s^{\prime}}$ analytically (assuming something about the three particle density functions), but due to the complicated nonlinear character of the equations, apart from some simple general statements, it is rather difficult to say anything about $d_{s s^{\prime}}$.

For initially homogeneous, relaxed dislocation systems $d_{s s^{\prime}}$ can be determined by DDD simulations. For this we do not have to study large systems (a few 100 dislocations is already enough), but we need several (around 1000) relaxed configurations to have the necessary statistics. Knowing the relaxed positions of dislocations $d_{s s^{\prime}}$ can be determined by simply counting the number of dislocation pairs at different relative positions.

Figure 11 shows the correlation function $d=d_{++}+d_{--}+d_{+-}+d_{-+}$obtained numerically. In the simulations parallel straight edge dislocations were considered at single slip geometry ( $\vec{b}$ is parallel to the $x$ axis). The number of dislocations was kept constant. Initially the dislocations were randomly distributed. By the numerical integration of Eq. (3.9) the relaxed dislocation configuration was determined at zero external stress.

The two most important properties of the correlation function are that next to the origin it is inversely proportional to the distance from the origin (analytical investigations revealed the same), and it decays to zero exponentially within a couple of average 


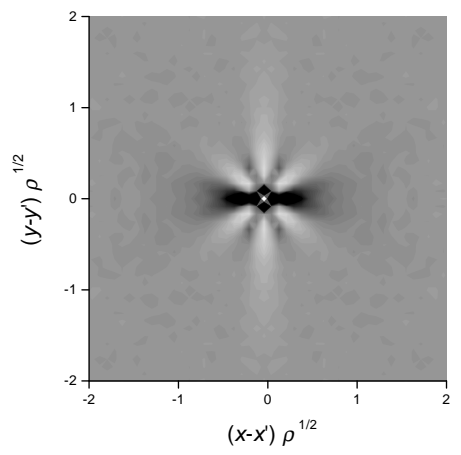

Figure 11. The total $d=d_{++}+d_{--}+d_{+-}+d_{-+}$dislocation-dislocation correlation function determined numerically at single slip geometry [61].

dislocation spacing. So, for originally random relaxed dislocation configurations the dislocation-dislocation correlation is short-ranged. With other words, if the distance of two dislocations is larger than a couple of times the average dislocation spacing the correlation between them is negligible [61,62]. We have to keep in mind, however, that this is valid only if the relaxed configuration is obtained from an initially random dislocation distribution. One can obviously set up initial configuration that relaxes to a strongly correlated state like for example a Taylor lattice. The problem is related to the constrained motion of dislocations. Since in the simulations only dislocation glide is allowed and dislocation multiplication is excluded, the number of dislocations in any narrow strip parallel to the Burgers vector cannot change during the relaxation of the system. It is determined by the initial configuration. The system does not "forget" fully the initial configuration. In reality, of course, the number of dislocations in a strip is determined by the dislocation multiplication. Since, however in 2D there is no "natural" law for dislocation multiplication one should investigate the correlation properties in 3D. Investigations are under their way, but at the moment we do not have conclusive results. Based on the results of $2 \mathrm{D}$ simulations in the following we assume that the correlation function is short range.

According to the results explained above it is plausible to assume that the correlation functions $d_{s s^{\prime}}\left(\vec{r}_{1}, \vec{r}_{2}\right)$ defined by the Eq. (3.54) can be approximated with the correlation function corresponding to a homogeneous system with dislocation density $\rho\left(\vec{r}_{1}\right)$. It follows that $d_{s s^{\prime}}\left(\vec{r}_{1}, \vec{r}_{2}\right)$ practically depends only on $\left(\vec{r}_{1}-\vec{r}_{2}\right)$, the direct $\vec{r}_{1}$ or $\vec{r}_{2}$ dependence is weak, it appears only through the spatial variation of the dislocation density, i.e.

$$
\rho_{s s^{\prime}}\left(\vec{r}_{1}, \vec{r}_{2}, t\right)=\rho_{s}\left(\vec{r}_{1}\right) \rho_{s^{\prime}}\left(\vec{r}_{2}\right)\left(1+d_{s s^{\prime}}\left(\vec{r}_{1}-\vec{r}_{2}\right)\right) \quad s, s^{\prime} \in\{+,-\}
$$


Similar approximation is used successfully for many other systems like for example in first principle quantum mechanics calculations to estimate the exchange energy. It is called "local density approximation".

By substituting Eq. (3.55) into Eqs. (3.30,3.31) after a long, but straightforward calculation we arrive at

$$
\begin{aligned}
& \frac{\partial \rho(\vec{r}, t)}{\partial t}+\vec{b} \frac{\partial}{\partial \vec{r}}\left[\kappa(\vec{r}, t)\left\{\tau_{s c}(\vec{r})+\tau_{\text {ext }}-\tau_{f}(\vec{r})+\tau_{b}(\vec{r})\right\}\right]=0 \\
& \frac{\partial \kappa(\vec{r}, t)}{\partial t}+\vec{b} \frac{\partial}{\partial \vec{r}}\left[\rho(\vec{r}, t)\left\{\tau_{s c}(\vec{r})+\tau_{\text {ext }}-\tau_{f}(\vec{r})+\tau_{b}(\vec{r})\right\}\right]=0,
\end{aligned}
$$

where

$$
\begin{gathered}
\tau_{f}(\vec{r})=\frac{1}{2} \int \rho\left(\vec{r}_{1}\right) d^{a}\left(\vec{r}-\vec{r}_{1}\right) \tau_{\text {ind }}\left(\vec{r}-\vec{r}_{1}\right) d \vec{r}_{1} \\
\tau_{b}(\vec{r})=\int \kappa\left(\vec{r}_{1}\right) d\left(\vec{r}-\vec{r}_{1}\right) \tau_{\text {ind }}\left(\vec{r}-\vec{r}_{1}\right) d \overrightarrow{r_{1}},
\end{gathered}
$$

in which the notations

$$
\begin{aligned}
d(\vec{r}) & =1 / 4\left[d_{++}(\vec{r})+d_{--}(\vec{r})+d_{+-}(\vec{r})+d_{-+}(\vec{r})\right] \\
d^{a}(\vec{r}) & =1 / 2\left[d_{+-}(\vec{r})-d_{-+}(\vec{r})\right]
\end{aligned}
$$

are introduced. Due to the following obvious symmetry properties of the correlation functions

$$
d_{+-}(\vec{r})=d_{-+}(-\vec{r}), \quad d_{++}(\vec{r})=d_{++}(-\vec{r}), \quad d_{--}(\vec{r})=d_{--}(-\vec{r})
$$

$d(\vec{r})$ is an even, while $d^{a}(\vec{r})$ is an odd function of $\vec{r}$. Furthermore, since the correlation functions correspond to a homogeneous system there is no other internal length scale but the average dislocation spacing $1 / \sqrt{\rho}$. It is obvious from simple dimensional analysis that the correlation functions depend only on the dimensionless quantity $\vec{r} \sqrt{\rho}$, i.e. $d(\sqrt{\rho} \vec{r})$ and $d^{a}(\sqrt{\rho} \vec{r})$.

Taking into account that the corelation functions decay to zero within a few dislocation spacing the fields $\kappa\left(\vec{r}_{1}\right)$ and $\rho\left(\vec{r}_{1}\right)$ appearing in Eqs. $(3.58,3.59)$ can be approximated by their Taylor expansion around the point $\vec{r}$. Keeping only the first nonvanishing terms we get that $[61,62]$

$$
\begin{gathered}
\tau_{f}(\vec{r})=\frac{\rho(\vec{r})}{2} \int d^{a}(\vec{r}) \tau_{\text {ind }}(\vec{r}) d \vec{r}, \\
\tau_{b}(\vec{r})=-\frac{\partial \kappa(\vec{r})}{\partial \vec{r}} \int \vec{r} d(\vec{r}) \tau_{\text {ind }}(\vec{r}) d \vec{r} .
\end{gathered}
$$

(To obtain expressions $(3.63,3.64)$ one has to take into account the symmetry properties of $d(\vec{r})$ and $d^{a}(\vec{r})$ explained above, and the relation $\tau_{\text {ind }}(\vec{r})=-\tau_{\text {ind }}(-\vec{r})$.) 
With the variable substitution $\vec{\eta}=\sqrt{\rho} \vec{r}, \tau_{f}(\vec{r})$ reads as

$$
\tau_{f}(\vec{r})=\frac{\sqrt{\rho(\vec{r})}}{2} \int d^{a}(\vec{\eta}) \tau_{i n d}(\vec{\eta}) d^{2} \vec{\eta} .
$$

where we took into account that $\tau_{\text {ind }}$ is proportional to $1 /|\vec{r}|$. By substituting the actual form of $\tau_{\text {ind }}$ given by Eq. (3.10) into Eq. (3.65) we get that

$$
\tau_{f}(\vec{r})=\frac{A C}{2} b \sqrt{\rho(\vec{r})},
$$

where $A=\mu /[2 \pi(1-\nu)]$ and

$$
C=\int \frac{\eta_{x}\left(\eta_{x}^{2}-\eta_{y}^{2}\right)}{\left(\eta_{x}^{2}+\eta_{y}^{2}\right)^{2}} d^{\prime a}\left(\eta_{x}, \eta_{y}\right) d \eta_{x} d \eta_{y} .
$$

in which the prime in $d^{\prime a}\left(\eta_{x}, \eta_{y}\right)$ indicates that $\vec{\eta}$ has to be measured in unit of average dislocation spacing. In order to see the physical meaning of the above expression, the external stress dependence of the parameter $C$ has to be analysed. The correlation function $d_{+-}$obviously varies with external stress (the equilibrium configuration of dislocation dipoles varies if stress is applied). Let us assume that the change of $d_{+-}$resulted by the external stress increases $C$. Since the change in $d_{-+}$is the opposite of the change of $d_{+-}$this causes also the increase of $C$ because of the minus sign in front of $d_{-+}$in the definition of $d^{a}$. As we see, the parameter $C$ depends on the external stress. Beside this, $\tau_{f}$ scales with $\sqrt{\rho}$. These support association of $\tau_{f}$ with the flow stress. Certainly, we have to be careful with this statement. In real dislocation systems hardening is caused by the forest dislocations which are not included into our model in any sense. Nevertheless, a stress like term showing similar properties as the flow stress appears naturally in the theory. The actual form of the stress dependence of $C$ is difficult to determine, but one can speculate that $\tau_{f}$ acts as static friction. It prevents dislocation motion, but it has a maximum scaling with $\sqrt{\rho}$.

After this let us analyse $\tau_{b}$ in more details. With the same variable substitution applied above we get that

$$
\tau_{b}(\vec{r})=-\frac{\partial \kappa(\vec{r})}{\partial \vec{r}} \frac{1}{\rho(\vec{r})} \int \vec{\eta} d^{\prime}(\vec{\eta}) \tau_{i n d}(\vec{\eta}) d^{2} \vec{\tau} .
$$

i.e.

$$
\tau_{b}(\vec{r})=-\frac{A D \vec{b}}{\rho} \frac{\partial \kappa(\vec{r})}{\partial \vec{r}}
$$

where

$$
D=\int \frac{\eta_{x}^{2}\left(\eta_{x}^{2}-\eta_{y}^{2}\right)}{\left(\eta_{x}^{2}+\eta_{y}^{2}\right)^{2}} d^{\prime}\left(\eta_{x}, \eta_{y}\right) d \eta_{x} d \eta_{y}
$$

is a dimensionless constant. In contrast with $C, D$ has only a weak external stress dependence because $d(\vec{r})$ contains the sum of the two correlation functions $d_{+-}$and 
$d_{-+}$changing oppositely. The actual value of $D$ can only be determined numerically. According to the numerical studies explained below it is in the order of magnitude of 1 .

With the results obtained above the evolution equations $(3.56,3.57)$ read as

$$
\begin{aligned}
& \frac{\partial \rho(\vec{r}, t)}{\partial t}+\vec{b} \frac{\partial}{\partial \vec{r}}\left[\kappa(\vec{r}, t)\left\{\tau(\vec{r}, t)-\tau_{f}-A D \frac{\vec{b}}{\rho(\vec{r}, t)} \frac{\partial \kappa(\vec{r}, t)}{\partial \vec{r})}\right\}\right]=f(\rho, \tau), \\
& \frac{\partial \kappa(\vec{r}, t)}{\partial t}+\vec{b} \frac{\partial}{\partial \vec{r}}\left[\rho(\vec{r}, t)\left\{\tau(\vec{r}, t)-\tau_{f}-A D \frac{\vec{b}}{\rho(\vec{r}, t)} \frac{\partial \kappa(\vec{r}, t)}{\partial \vec{r}}\right\}\right]=0 .
\end{aligned}
$$

where $\tau=\tau_{s c}+\tau_{\text {ext }}$ is the total macroscopic stress.

From Eq. (3.36) the constitutive equation of the plastic shear rate can also be given as

$$
\dot{\gamma}=b^{2} \rho(\vec{r}, t)\left\{\tau(\vec{r}, t)-\tau_{f}-A D \frac{\vec{b}}{\rho(\vec{r}, t)} \frac{\partial \kappa(\vec{r}, t)}{\partial \vec{r}}\right\} .
$$

With Eq. (3.34)

$$
\dot{\gamma}=b^{2} \rho(\vec{r}, t)\left\{\tau(\vec{r}, t)-\tau_{f}-A D \frac{1}{b^{2} \rho(\vec{r}, t)}\left(\vec{b} \frac{\partial}{\partial \vec{r}}\right)^{2} \gamma(\vec{r}, t)\right\} .
$$

If we introduce the effective stress

$$
\tau_{e f f}(\vec{r})=\tau(\vec{r})-A D \frac{1}{b^{2} \rho(\vec{r})}\left(\vec{b} \frac{\partial}{\partial \vec{r}}\right)^{2} \gamma(\vec{r})
$$

it looks similar to the effective stress (1.2) suggested by E. Aifantis from phenomenological considerations. An important difference, however, is that in Eq. (3.75) the length scale $l=1 / \sqrt{\rho}$ appearing in front of the gradient term is a natural one, it is not a material parameter suggested in the phenomenological non-local continuum theories. The length scale $1 / \sqrt{\rho}$ obeys an evolution equation (Eq. (refrhof).

\subsection{Deformation of a constrained channel}

To illustrate some implications of the evolution equations derived in the previous subsection we study a very simple example, namely a constrained channel deforming in simple shear as shown in Figure 12 [62]. A channel of width $L$ in the $x$ direction and infinite extension in the $y$ direction is bounded by walls that are impenetrable for dislocations (i.e., the plastic deformation in the walls is zero). The slip direction corresponds to the $x$ direction, and the layer is sheared by a constant shear stress $\tau_{\text {ext }}$. The whole assembly is embedded in an infinite crystal.

The system envisaged is particularly simple because it is homogeneous in the $y$ direction (the dislocation densities depend on the coordinate $x$ in the slip direction only). It follows from Eq. (3.41) that in this case the long-range self-consistent stress field is zero for an arbitrary function $\kappa(x)$, i.e., any dislocation interactions in the system are of 


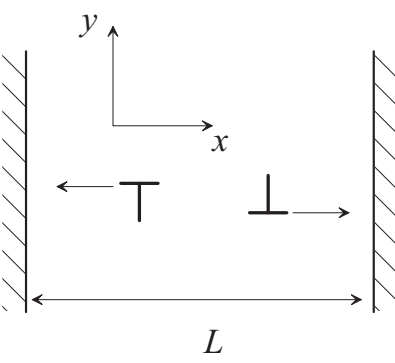

Figure 12. Geometry of a constrained channel

short-range nature and hence described by the flow stress $\tau_{f}$ and the gradient-dependent stress $\tau_{b}$.

Before investigating the behaviour resulting from Eqs. (3.71) and (3.72) and comparing it with the results obtained from discrete simulations, it is instructive to have a look at the results we get from the mean-field model defined by Eqs. (3.38) and (3.39). Since the self-consistent stress is zero, the mean-field model becomes trivial: Whatever the initial conditions, for an arbitrarily small, positive value of the external stress all positive dislocations 'condense' at the right wall and all negative dislocations at the left one. For an initially homogeneous dislocation distribution with density $\rho_{0}$, the strain achieved by this condensation is $\gamma_{\infty}=\rho_{0} b L / 2$. Hence, the system exhibits a trivial size effect (the achievable strain is proportional to the size of the system, which determines the mean dislocation path). However, as demonstrated in the following, the prediction that this strain is achieved at arbitrarily small external stress is grossly unrealistic.

We now revert to the gradient-dependent model derived in the previous subsection. We assume an initially homogeneous dislocation distribution of density $\rho_{0}$. To facilitate comparison with discrete simulations, it is convenient to introduce scaled stress, space and dislocation density variables through $\tau=A b \sqrt{\rho_{0}} \tilde{\tau}, x=D \tilde{x} / \sqrt{\rho}, \rho=\rho_{0} \tilde{\rho}$, and $\kappa=\rho_{0} \tilde{\kappa}$. In scaled variables and after corresponding re-scaling of time, Eqs. (3.71) and (3.72) read

$$
\begin{aligned}
& \partial_{t} \tilde{\rho}(\tilde{x}, t)=-\partial_{\tilde{x}}\left[\tilde{\kappa}(\tilde{x}, t)\left\{\tilde{\tau}-[1 / \tilde{\rho}(\tilde{x}, t)] \partial_{\tilde{x}} \tilde{\kappa}(\tilde{x}, t)\right\}\right], \\
& \partial_{t} \tilde{\kappa}(\tilde{x}, t)=-\partial_{\tilde{x}}\left[\tilde{\rho}(\tilde{x}, t)\left\{\tilde{\tau}-[1 / \tilde{\rho}(\tilde{x}, t)] \partial_{\tilde{x}} \tilde{\kappa}(\tilde{x}, t)\right\}\right] .
\end{aligned}
$$

To formulate the boundary conditions at the walls located at $\tilde{x}= \pm \tilde{L} / 2$, we note that no dislocations can enter the system through the walls. Hence, the density of positive dislocations (moving to the right) at the left wall and the density of negative dislocations at the right wall are zero, i.e. $\tilde{\kappa}(-\tilde{L} / 2)=-\tilde{\rho}(-\tilde{L} / 2), \tilde{\kappa}(\tilde{L} / 2)=\tilde{\rho}(\tilde{L} / 2)$. Furthermore, the dislocation fluxes at the walls must be zero, which requires that $\left[\tilde{\rho} \tilde{\tau}-\partial_{\tilde{x}} \tilde{\kappa}\right]=0$ at $\tilde{x}= \pm \tilde{L} / 2$.

The initial conditions are $\tilde{\rho}(\tilde{x}, 0)=1$ and $\tilde{\kappa}(\tilde{x}, 0)=0$ everywhere except directly at the walls where we assume non-zero values of $\kappa$ in a narrow boundary layer to satisfy the boundary conditions. We make the simplifying assumption that the effective stress can be 
represented as the external stress diminished by the (spatially homogeneous) flow stress of an infinite system, and perform a 'deformation experiment' as follows: we increase the effective stress from zero in an adiabatically slow manner, i.e., after each small stress increment the system is allowed to relax until it reaches a stationary configuration. After this relaxation, the scaled strain is calculated as $\tilde{\gamma}=-\int \tilde{\kappa} d \tilde{x}$ (see Eq. (3.34)), then the stress is increased again, etc. The resulting stress-strain curves for different values of $L$ are compiled in Figure 13 [62]. It can be seen that the behaviour is very different from

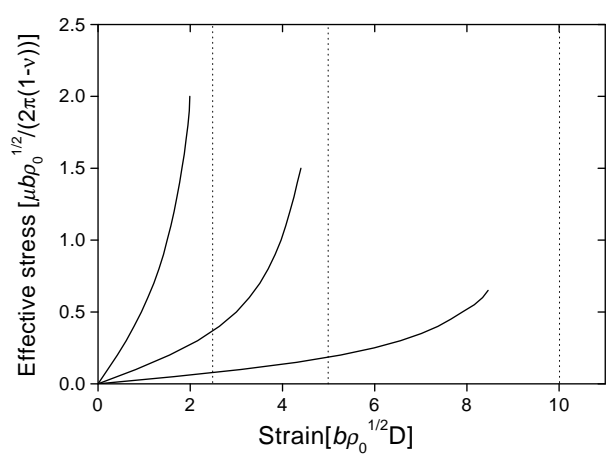

Figure 13. Stress-strain curves obtained at different channel size $L$.

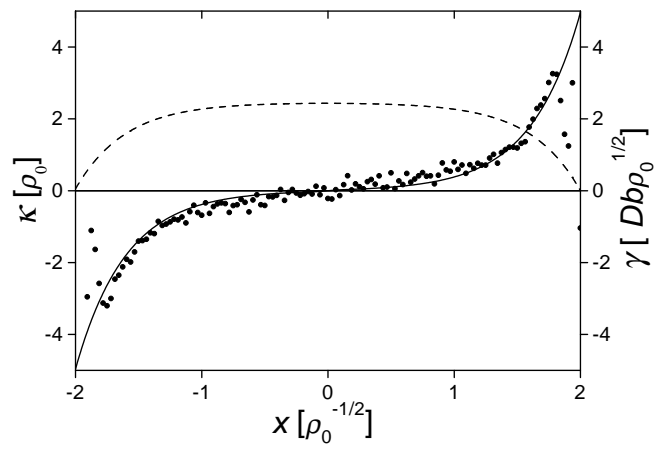

Figure 14. Signed dislocation density and strain profiles for a system of width $L=$ $4 / \sqrt{\rho_{0}}$ at scaled stress $\tilde{\tau}=2 ; \kappa(x)$ from discrete simulation (data points), $\kappa(x)$ from continuum model (full line), strain profile from continuum model (dashed line).

the prediction of the mean-field model: the strain increases gradually with stress and reaches the limit strain $\gamma_{\infty}\left(\tilde{\gamma}_{\infty}=\tilde{L} / 2\right.$ in scaled units) only asymptotically. In physical terms this behaviour stems from the fact that there is a short-range repulsion between 
individual dislocations of the same sign as they pile up against the walls (see Figure 14). To increase the strain towards the asymptotic strain, this repulsion must be overcome, which requires an increasing stress that diverges as $\gamma \rightarrow \gamma_{\infty}$.

Looking at the distribution of dislocation densities and strains within the channel, we find that at high stresses two boundary layers emerge near the walls (Figure 14). Its properties can be analysed by the equilibrium condition

$$
\tau_{\text {ext }}-A D \frac{\vec{b}}{\rho(\vec{r}, t)} \frac{\partial \kappa(\vec{r}, t)}{\partial \vec{r}}=0
$$

required to hold at steady state. (Since, for the geometry considered the self-consistent field is zero, $\left.\tau=\tau_{\text {ext }}\right)$. Near the boundaries most of the dislocations have the same sign, $\rho \approx|\kappa|$. According to this, near the left boundary Eq. (3.78) reads as

$$
\tau_{e x t} \kappa=-A D b \frac{d \kappa(x)}{d x}
$$

with solution

$$
\kappa(x)=\kappa_{0} \exp \left\{-\frac{\tau_{e x t}}{A D b} x\right\} .
$$

(A similar expression obviously holds near the right side, too.) As it is seen, the width of the boundary layers decreases with increasing external stress.

It is interesting to compare this result with the prediction of the phenomenological gradient approach. According to Eq. (1.2) for the problem considered the equilibrium condition is

$$
\tau_{\text {ext }}-\frac{\mu}{l^{2}} \frac{\kappa(x)}{d x}=0
$$

with solution

$$
\kappa(x)=\kappa_{0}+\frac{l^{2}}{\mu} \tau_{e x t} x
$$

In the centre part of the channel, where $\rho$ is nearly constant, the linear relation predicted by the phenomenological gradient approach describes well the observed variation of $\kappa$, but it is not able to account for the boundary layers.

The results obtained are compared with DDD simulations performed on the same system. To get reliable statistics, stress-strain graphs and the corresponding dislocation density profiles were averaged over a huge ensemble (typically several thousands of simulations). An example of a dislocation density profile obtained from this procedure is illustrated in Figure 14 which shows a $\tilde{\kappa}(\tilde{x})$ profile averaged over 2000 simulations of systems with length $L=4 / \sqrt{\rho_{0}}$ and (periodically repeated) height $16 / \sqrt{\rho_{0}}$. The profile shown in the figure has been taken at a scaled effective stress $\tilde{\tau}=2$. It is seen that indeed two boundary layers emerge. From the width of these boundary layers we can directly determine the constant $D$ for the present type of simulation, which turns out to be $D=0.8$. Using the continuum model with this value of $D$ yields the full line 


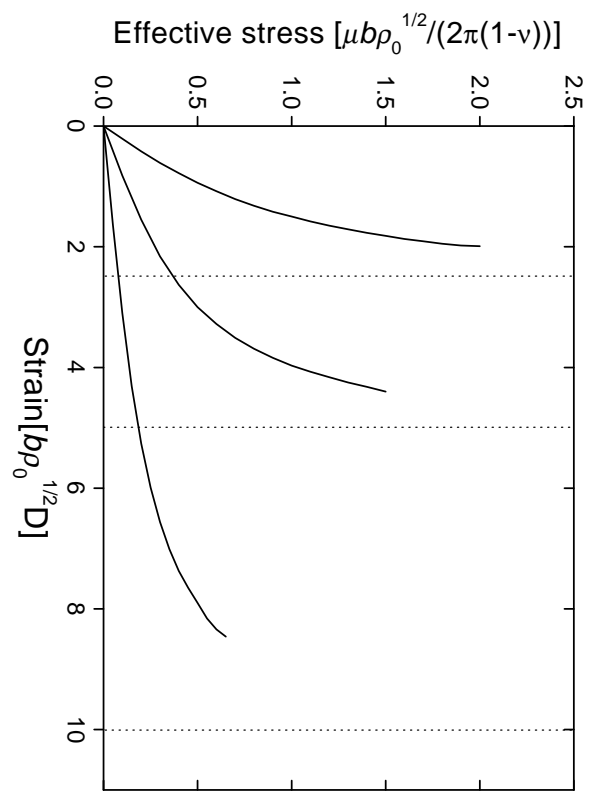

Figure 15. Comparison of stress-strain graphs; full line continuum model; data points: discrete simulation. Parameters are the same as in Figure 14.

in Figure 14, which shows that the density profile obtained from the continuum model matches well the discrete simulation except in the immediate vicinity of the walls.

As seen from Figure 15, also the stress-strain curves for the discrete and continuum models exhibit almost perfect agreement. By varying the system size and initial dislocation density, we find that, for sufficiently high stresses the width of the boundary layers at fixed stress $\tau_{\text {ext }}$ is within the error margins indeed independent on the system size and the dislocation density. If the applied stress is increased, the boundary layer width is found to decrease. Again all these findings are in line with the predictions of the continuum model.

\subsection{Application to metal-matrix composite}

In order to demonstrate the capability of the continuum theory of dislocations explained above we shortly summarise the simulation results obtained on a $2 \mathrm{D}$ model system of a metal-matrix composite [54,63]. It contains rigid rectangular particles arranged in a hexagonal packing, as illustrated in Figure 16. The cell is subjected to plane strain, simple shear, which is prescribed through the boundary conditions

$$
u_{1}= \pm h \Gamma \quad, \quad u_{2}=0 \quad \text { along } x_{2}= \pm h,
$$




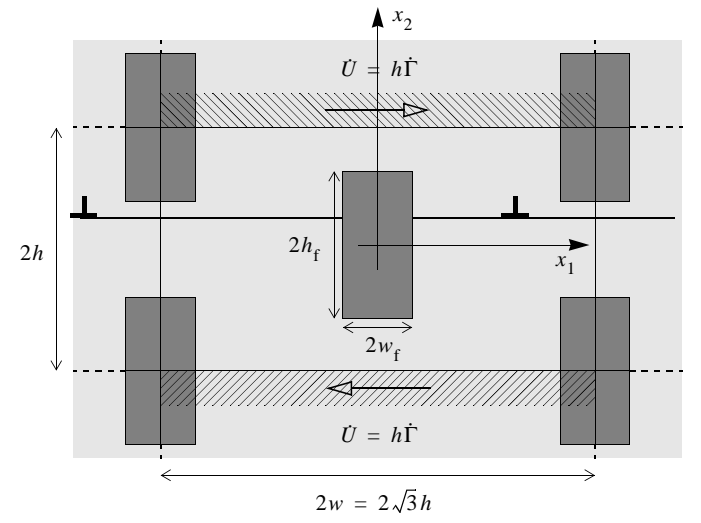

Figure 16. Unit cell in a doubly-periodic array of elastic particles, subjected to simple shear. The slip planes are taken to be parallel to the shear direction $(x)$.

where $\Gamma$ is the applied shear. Periodic boundary conditions are imposed along the lateral sides $x= \pm w$. The slip plane normal $\vec{n}$ is in the $y$-direction and the Burgers vector is parallel to the $x$-direction. Two reinforcement morphologies were analysed having the same area fraction of $20 \%$ but different geometric arrangements of the reinforcing phase. In one morphology, material (i), the particles are square and are separated by unreinforced veins of matrix material while in the other, material (iii), the particles are rectangular and do not leave any unreinforced veins of matrix material.

The problem was studied in details by Cleveringa et al. [54, 63] with DDD simulation. Here we compare their DDD simulation results with the results obtained by solving Eqs. $(3.71,3.72)$ with a finite element method (for the details of the numerical technique used see $[64,65])$. For the dislocation density field equations, boundary conditions need to be specified at the boundary of the cell as well as along the interface with the elastic particles. Along the cell sides $x= \pm w$, periodic boundary conditions are applied, while along $y= \pm h$ we have the natural condition that there is no flux of dislocations across these boundaries. Similar conditions apply along the top and bottom interfaces with the particles. Along the vertical sides of the particles, we impose that the slip rate vanishes.

As it is seen in Figure 17 the stress-strain curves obtained by DDD simulation and from the continuum theory match extremely well for both reinforcement morphologies investigated [64, 65]). Figure 18 shows the $\rho$ and $\kappa$ maps obtained for the (iii) morphology. It can be seen that, like at the shear of the channel discussed above, a boundary layer of geometrically necessary dislocations develops at the vertical unpenetrable surface of the composite particles.

In conclusion it can be stated that for single slip the continuum theory developed predicts the same behaviour as the DDD simulations. How to generalise the continuum model to multiple slip is being studied intensively [66, 67]. At the moment the theory is 


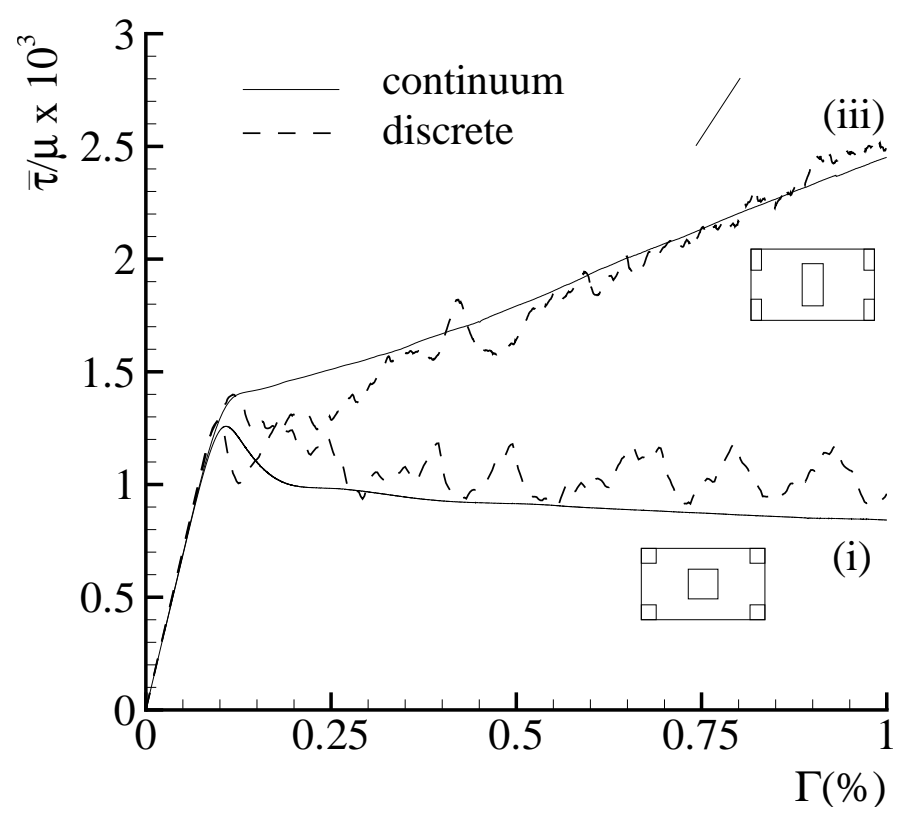

Figure 17. Stress-stain curves obtained by DDD simulations (dashed lines) and by solving the continuum Eqs. $(3.71,3.72)$ (full line).

far from being fully developed. It is even more difficult to extend the statistical approach to $3 \mathrm{D}$. In order to show the possible approaches, in the next part we shortly summarise two $3 \mathrm{D}$ continuum models proposed recently.

\subsection{Boltzmann theory of dislocations}

To describe the evolution of dislocation loops El-Azab has recently proposed a new theoretical framework [68], which is the generalisation of the Boltzmann equation for line type objects. In his model only planar loops are considered, i.e. dislocation climb is excluded.

In contrast with point like particles (considered in Boltzmann's original theory), beside its position a dislocation segment has another degree of freedom, its line direction $\vec{l}$. In a plane the line direction $\vec{l}$ can be represented with a scalar parameter $\Theta$ which is the angle between $\vec{l}$ and a given direction in the plane. According to this, the probability density function $f$ is the function of $\vec{r}, \vec{v}$ and $\Theta$. (To avoid the problems related to define the mass of a dislocation, instead of the moment the velocity of the dislocation segment is used to give the state of the segment.) Since, as it is explained earlier, the probability density function is system size dependent ( $f$ is normalised to 1 ) it is more convenient 

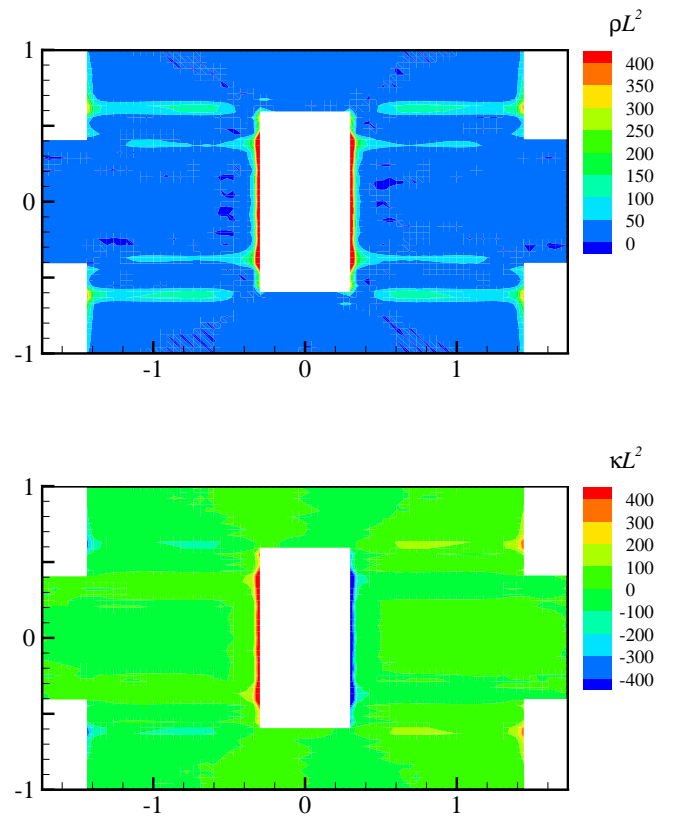

Figure 18. $\rho$ (top box) and $\kappa$ (bottom box) maps at $1 \$$ deformation for the (iii) morphology.

to operate with dislocation line length density $\phi$. Hence, $\phi^{(i)}(\vec{r}, \vec{v}, \Theta, t)$ can be given the definition: $\phi^{(i)}(\vec{r}, \vec{v}, \Theta, t) d^{3} \vec{r} d^{3} \vec{v} d \Theta$ is the dislocation line length with Burgers vector $\vec{b}_{i}$ $(i=1 . . N)$ in the $d^{3} \vec{r} d^{3} \vec{v} d \Theta$ phase space at time $t$. It has to be noted that since $\vec{v}$ is perpendicular to $\vec{l}, \dot{\Theta}$ is not an "independent" variable, that is why it does not appear among the arguments of $\phi^{(i)}$.

The conventional field variables are now derived from $\phi^{(i)}$. The dislocation line density is defined as follows:

$$
\rho(\vec{r}, t)=\sum_{i=1}^{N} \rho^{(i)}(\vec{r}, t)
$$

where

$$
\rho^{(i)}(\vec{r}, t)=\iint \phi^{(i)}(\vec{r}, \vec{v}, \Theta, t) d^{3} \vec{v} d \Theta
$$


According to Eq. (2.13) the dislocation density tensor $\hat{\alpha}$ is given by

$$
\alpha_{m n}(\vec{r}, t)=\sum_{i=1}^{N} \alpha_{m n}^{(i)}(\vec{r}, t)
$$

where

$$
\alpha_{m n}^{(i)}(\vec{r}, t)=\iint l_{m}(\Theta) b_{n}^{(i)} \phi^{(i)}(\vec{r}, \vec{v}, \Theta, t) d^{3} \vec{v} d \Theta
$$

while from Eq. (2.40) the dislocation current density $\hat{j}$ reads as

$$
j_{m n}(\vec{r}, t)=\sum_{i=1}^{N} j_{m n}^{(i)}(\vec{r}, t)
$$

where

$$
j_{n k}^{(i)}(\vec{r}, t)=\iint e_{n m p} l_{m}(\Theta) v_{p} b_{k}^{(i)} \phi^{(i)}(\vec{r}, \vec{v}, \Theta, t) d^{3} \vec{v} d \Theta .
$$

Taking into account that the velocity of a dislocation segment is perpendicular to the line direction, we can conclude that the evolution equation of $\phi^{(i)}$ has the form (for the details of the derivation see [68]):

$$
\left(\frac{\partial}{\partial t}+v_{j} \frac{\partial}{\partial x_{j}}+\dot{v}_{j}(\hat{\alpha}, \hat{\sigma}, . .) \frac{\partial}{\partial v_{j}}\right) \phi^{(i)}(\vec{r}, \vec{v}, \Theta, t)=s^{(i)}(\vec{r}, \vec{v}, \Theta), \quad i=1, . ., N
$$

where $s^{(i)}(\vec{r}, \vec{v}, \Theta)$ represents all the possible source terms, and $\dot{v}_{j}(\hat{\alpha}, \hat{\sigma}, .$.$) stands for the$ acceleration of the dislocation. which is a function of the different fields like $\hat{\alpha}$ and $\hat{\sigma}$. A remarkable feature of the above equation is that the derivative with respect to $\Theta$ does not appear in the left hand side.

If we multiply the above equation with $l_{m}(\Theta) b_{n}^{(i)}$ and integrate it with respect to the velocity and $\Theta$, after long but straightforward algebraic manipulations we conclude:

$$
\alpha_{m n}^{(i)}+e_{m k l} \frac{\partial}{\partial r_{k}} j_{l n}^{(i)}=S_{n m}^{(i)},
$$

where

$$
S_{n m}^{(i)}=\iint l_{n}(\Theta) b_{m}^{(i)} s^{(i)}(\vec{r}, \vec{v}, \Theta) d^{3} \vec{v} d \Theta .
$$

By summing up Eq. (3.91) over all Burgers vectors and comparing the result with Eq. (2.37) we obtain an important condition the source terms have to fulfill

$$
\sum_{i=1}^{N} S_{n m}^{(i)}=0
$$


To get a closed equation for the evolution of $\phi^{(i)}(\vec{r}, \vec{v}, \Theta, t)$, the actual forms of $s^{(i)}(\vec{r}, \vec{v}, \Theta)$ and $\dot{v}_{j}(\hat{\alpha}, \hat{\sigma}, .$.$) should be given. At the present stage of the theory these$ are not well established.

We mention that it has been proposed recently by Hochrainer and Zaiser [69] that beside $\Theta$ the curvature of the dislocation line should also be considered as an independent variable. This may help setting up the constitutive relations.

\subsection{Hydrodynamics approach proposed by Kratochvíl and Sedláček}

In hydrodynamics the state of the material is described by the density $\rho(\vec{r}, t)$ and the velocity $\vec{u}(\vec{r}, t)$ fields. They are the appropriate mean values of the probability density function $f(\vec{r}, \vec{p}, t)$. One may operate with the same quantities in dislocation theory. However, to account for the line direction degree of freedom an additional field denoted by $\beta(\vec{r}, t)$ has to be introduced. The three fields can be formally defined as

$$
\begin{aligned}
\rho(\vec{r}, t) & =\iint \phi(\vec{r}, \vec{v}, \Theta, t) d \vec{v} d \Theta \\
\vec{u}(\vec{r}, t) & =\frac{1}{\rho(\vec{r}, t)} \iint \vec{v} \phi(\vec{r}, \vec{v}, \Theta, t) d \vec{v} d \Theta \\
\beta(\vec{r}, t) & =\frac{1}{\rho(\vec{r}, t)} \iint \Theta \phi(\vec{r}, \vec{v}, \Theta, t) d \vec{v} d \Theta,
\end{aligned}
$$

where for the sake of simplicity we assumed that all dislocation loops have the same Burgers vector (the superscript $(i)$ is dropped out) and their slip planes are parallel.

Taking the $z$ axis perpendicular to the slip plane of the loops, the two nonvanishing components of the dislocation density tensor are

$$
\alpha_{11}=b \rho \cos (\beta), \quad \alpha_{21}=b \rho \sin (\beta) .
$$

Since the dislocation density tensor is the curl of the plastic distortion, it has to be div free:

$$
\frac{\partial \alpha_{i j}}{\partial r_{i}}=0 .
$$

One can find from Eqs $(3.97,3.98)$ that $\rho$ and $\beta$ have to satisfy the conservation law

$$
\frac{\partial \rho \cos (\beta)}{\partial x}+\frac{\partial \rho \sin (\beta)}{\partial y}=0 .
$$

On the other hand, from the general expression of the evolution of the dislocation density tensor given by Eq. (2.37), the following evolution equations can be deduced for $\alpha$ and $\beta$ fields(for details see [70])

$$
\begin{aligned}
\rho \dot{\beta} & =\cos (\beta) \frac{\partial \rho v}{\partial x}+\sin (\beta) \frac{\partial \rho v}{\partial y}, \\
\dot{\rho} & =\sin (\beta) \frac{\partial \rho v}{\partial x}-\cos (\beta) \frac{\partial \rho v}{\partial y},
\end{aligned}
$$


where $v=|\vec{v}|$.

To have a closed theory a constitutive relation is needed between the three fields $\rho(\vec{r}, t), \beta(\vec{r}, t)$ and $v(\vec{r}, t)$. Kratochvíl and Sedláček [70] suggested the following constitutive relation for the velocity field :

$$
B v=\left\{\begin{array}{lll}
b \sigma_{13}+C \kappa_{s}-b \tau_{0}-b \tau & \text { if } & b \sigma_{13}+C \kappa_{s}>b \tau_{0}+b \tau \\
0 & \text { if } & \left|b \sigma_{13}+C \kappa_{s}\right|<b \tau_{0}+b \tau \\
b \sigma_{13}+C \kappa_{s}+b \tau_{0}+b \tau & \text { if } & b \sigma_{13}+C \kappa_{s}<-b \tau_{0}-b \tau
\end{array}\right.
$$

where $b \sigma_{13}$ is the Peach-Koehler force due to the local shear stress, $C \kappa_{s}$ is the self-force, $b \tau_{0}$ is the friction force, and $b \tau$ represents the interaction between the gliding dislocations an the dislocation loops.

The self-force $C \kappa_{s}$ is considered in the line tension approximation, where $\kappa_{s}$ is the dislocation line tension. The curvature of a dislocation segment $C(\vec{r}, \beta, t)=-\operatorname{div} \vec{n}$, where $\vec{n}$ is the unit normal to the dislocation segment. As it is explained in details in [70] $C$ can be approximated by the expression

$$
C=\cos (\beta) \frac{\partial \beta}{\partial x}+\sin (\beta) \frac{\partial \beta}{\partial y}
$$

The most difficult problem is to set up an appropriate expression for $b \tau$. For this Kratochvíl and Sedláček [70] suggested that

$$
b \tau=F c^{1 / 3}
$$

were $c$ is the loop density, and $F$ is a constant.

According to detailed analytical and numerical investigations [70] the model explained above is able to predict both dislocation patterning and size effect. Nevertheless, the justification of the assumptions used requires further investigation.

\section{Internal stress distribution generated by the dislocations}

In order to describe the statistical properties of a dislocation ensemble, a key quantity is the internal stress distribution. In this section the general form of the probability distribution of the internal shear stress $P(\tau)$ is determined for a system of parallel edge dislocations. As it is discussed later the results can be generalised to 3D systems.

\subsection{General considerations}

Like in the previous section, let us consider a system of $N$ parallel straight edge dislocations positioned at the points $\vec{r}_{i}, i=\overline{1, N}$ in the $x y$ plane perpendicular to the dislocation lines. For the sake of simplicity, we assume that each dislocation has the same Burgers vector $\vec{b}$. As it is shown later, the generalisation of the results for systems consisting of dislocations with different Burgers vectors is straightforward [71].

The internal shear stress at the point $\vec{r}$ is the sum of the stress fields of the individual dislocations

$$
\tau(\vec{r})=\sum_{i=1}^{N} \tau_{i n d}\left(\vec{r}-\vec{r}_{i}\right) .
$$


In the first part of our analysis we assume that there is no dislocation at the point $\vec{r}$. Later on, the stress distribution at the dislocations is discussed, too.

The precise definition of the problem addressed in this section is to determine the $P\left(\tau_{0}\right) d \tau_{0}$ probability of occurrence of $\tau$ in the range

$$
\tau_{0}-\frac{d \tau_{0}}{2} \leq \tau(\vec{r}) \leq \tau_{0}+\frac{d \tau_{0}}{2}
$$

where $\tau_{0}$ is a preassigned value for $\tau . P\left(\tau_{0}\right)$ can be obtained as a direct application of Markoff's method [72] applied for several problems, like the problem of random flights, or for the determination of the distribution of forces in gravitationally interacting random systems. In contrast with the two problem mentioned, for dislocations the $N$ particle distribution function cannot be built up from the one particle distribution functions since as it will be shown later it would lead to system size dependent internal stress distribution function $P\left(\tau_{0}\right)$. To avoid this, dislocation-dislocation correlation must be taken into account.

Denoting the $N$ particle dislocation density function by $f_{N}\left(\vec{r}_{1}, \vec{r}_{2}, \ldots, \vec{r}_{N}\right)$ the internal stress distribution can be expressed as

$$
P\left(\tau_{0}\right) d \tau_{0}=\int \ldots \int f_{N}\left(\vec{r}_{1}, \vec{r}_{2}, \ldots, \vec{r}_{N}\right) d \vec{r}_{1} d \vec{r}_{2} \ldots d \vec{r}_{N},
$$

where the integration is effected only over that part of configuration space for which the inequalities (4.2) are satisfied. By the introduction of the factor

$$
\Delta\left(\vec{r}_{1}, \vec{r}_{2}, \ldots, \vec{r}_{N}\right)=\left\{\begin{array}{ll}
1 & \text { whenever } \\
0 & \text { otherwise }
\end{array} \tau_{0}-\frac{d \tau_{0}}{2} \leq \tau \leq \tau_{0}+\frac{d \tau_{0}}{2}\right.
$$

the integral in Eq. (4.3) can be extended over $2 N$ dimensional space $\Re^{2 N}$ :

$$
P\left(\tau_{0}\right) d \tau_{0}=\int_{-\infty}^{\infty} \ldots \int_{-\infty}^{\infty} \Delta\left(\vec{r}_{1}, \vec{r}_{2}, \ldots, \vec{r}_{N}\right) f_{N}\left(\vec{r}_{1}, \vec{r}_{2}, \ldots, \vec{r}_{N}\right) d \vec{r}_{1} d \vec{r}_{2} \ldots d \vec{r}_{N} .
$$

For the determination on the structure of expression (4.5) one has to consider the integral

$$
\delta=\frac{1}{\pi} \int_{-\infty}^{\infty} \frac{\sin (\alpha q)}{q} \exp [i \gamma q] d q
$$

which is the well-known discontinuous integral of the Dirichlet function with the properties

$$
\delta=\left\{\begin{array}{lll}
1 & \text { whenever } & -\alpha<\gamma<\alpha \\
0 & \text { otherwise }
\end{array}\right.
$$

By taking

$$
\alpha=\frac{d \tau_{0}}{2} \quad \text { and } \quad \gamma=\sum_{i=1}^{N} \tau_{i n d}\left(\vec{r}-\vec{r}_{i}\right)-\tau_{0}
$$


from Eq. (4.4) one gets that $\Delta=\delta$. With the substitution of the (4.6) form of $\delta$ into Eq. (4.5), we obtain that

$$
\begin{aligned}
P\left(\tau_{0}\right) d \tau_{0} & =\frac{1}{\pi} \int_{\Re} d q \int_{\Re^{2 N}} d \vec{r}_{1} d \vec{r}_{2} \ldots d \vec{r}_{N} f_{N}\left(\vec{r}_{1}, \vec{r}_{2}, \ldots, \vec{r}_{N}\right) \\
& \times \frac{\sin \left(\frac{1}{2} d \tau_{0} q\right)}{q} \exp \left\{i\left[\sum_{i=1}^{N} \tau_{i n d}\left(\vec{r}-\vec{r}_{i}\right) q-\tau_{0} q\right]\right\} .
\end{aligned}
$$

It can be seen from the structure of the above expression that the Fourier transform of the internal stress distribution

$$
A_{N}(\vec{r}, q)=\bar{P}\left(\tau_{0}\right)
$$

has the form

$$
A_{N}(\vec{r}, q)=\int f_{N}\left(\vec{r}_{1}, \vec{r}_{2}, \ldots, \vec{r}_{N}\right) \prod_{j=1}^{N} \exp \left\{i q \tau\left(\vec{r}-\vec{r}_{j}\right)\right\} d^{2} \vec{r}_{1} d^{2} \vec{r}_{2} \ldots d^{2} \vec{r}_{N} .
$$

If we introduce the function

$$
B(\vec{r}, q)=1-\exp \left\{i \tau_{\text {ind }}(\vec{r}) q\right\}
$$

expression (4.11) can be rewritten into a power series of $B(\vec{r}, q)$

$$
\begin{aligned}
A_{N}(\vec{r}, q)= & \int d \vec{r}_{1} d \vec{r}_{2} \ldots d \vec{r}_{N} f_{N}\left(\vec{r}_{1}, \vec{r}_{2}, \ldots, \vec{r}_{N}\right) \prod_{j=1}^{N}\left[1-B\left(\vec{r}-\vec{r}_{j}, q\right)\right] \\
= & 1-\int \rho_{1}\left(\vec{r}_{1}\right) B\left(\vec{r}-\vec{r}_{1}, q\right) d^{2} \vec{r}_{1} \\
& +\frac{1}{2} \int \rho_{2}\left(\vec{r}_{1}, \vec{r}_{2}\right) B\left(\vec{r}-\vec{r}_{1}, q\right) B\left(\vec{r}-\vec{r}_{2}, q\right) d^{2} \vec{r}_{1} d^{2} \vec{r}_{2}+\ldots
\end{aligned}
$$

where $\rho_{k}\left(\vec{r}_{1}, \vec{r}_{2}, \ldots, \vec{r}_{k}\right)$ is the $k$-th order dislocation-density function defined by Eq. (3.15). Eq. (4.13) can be transformed into an exponential form

$$
A_{N}(\vec{r}, q)=\exp \{E(q, \vec{r})\}
$$

where

$$
\begin{aligned}
E(q, \vec{r})= & -\int \rho_{1}\left(\vec{r}_{1}\right) B\left(\vec{r}-\vec{r}_{1}, q\right) d^{2} \vec{r}_{1} \\
& +\frac{1}{2} \int D_{2}\left(\vec{r}_{1}, \vec{r}_{2}\right) B\left(\vec{r}-\vec{r}_{1}, q\right) B\left(\vec{r}-\vec{r}_{2}, q\right) d^{2} \vec{r}_{1} d^{2} \vec{r}_{2}+\ldots
\end{aligned}
$$

in which

$$
D_{2}\left(\vec{r}_{1}, \vec{r}_{2}\right)=\rho_{2}\left(\vec{r}_{1}, \vec{r}_{2}\right)-\rho_{1}\left(\vec{r}_{1}\right) \rho_{1}\left(\vec{r}_{2}\right)=\rho_{1}\left(\vec{r}_{1}\right) \rho_{1}\left(\vec{r}_{2}\right)\left(1+d\left(\vec{r}_{1}, \vec{r}_{2}\right)\right)
$$


is the dislocation-dislocation correlation function.

Eq. (4.15) is straightforward to generalise for the case where dislocations with Burgers vectors $\pm \vec{b}$ are allowed. One can find that

$$
\begin{aligned}
E(q, \vec{r})= & -\int\left[\rho_{+}\left(\vec{r}_{1}\right) B\left(\vec{r}-\vec{r}_{1}, q\right)+\rho_{-}\left(\vec{r}_{1}\right) B^{*}\left(\vec{r}-\vec{r}_{1}, q\right)\right] d^{2} \vec{r}_{1} \\
& +\frac{1}{2} \int D_{++}\left(\vec{r}_{1}, \vec{r}_{2}\right) B\left(\vec{r}-\vec{r}_{1}, q\right) B\left(\vec{r}-\vec{r}_{2}, q\right) d^{2} \vec{r}_{1} d^{2} \vec{r}_{2} \\
& +\frac{1}{2} \int D_{+-}\left(\vec{r}_{1}, \vec{r}_{2}\right) B\left(\vec{r}-\vec{r}_{1}, q\right) B^{*}\left(\vec{r}-\vec{r}_{2}, q\right) d^{2} \vec{r}_{1} d^{2} \vec{r}_{2} \\
& +\frac{1}{2} \int D_{--}\left(\vec{r}_{1}, \vec{r}_{2}\right) B^{*}\left(\vec{r}-\vec{r}_{1}, q\right) B^{*}\left(\vec{r}-\vec{r}_{2}, q\right) d^{2} \vec{r}_{1} d^{2} \vec{r}_{2} \\
& +\frac{1}{2} \int D_{-+}\left(\vec{r}_{1}, \vec{r}_{2}\right) B^{*}\left(\vec{r}-\vec{r}_{1}, q\right) B\left(\vec{r}-\vec{r}_{2}, q\right) d^{2} \vec{r}_{1} d^{2} \vec{r}_{2}+\ldots
\end{aligned}
$$

where $" * "$ denotes complex conjugate.

\subsection{Stress distribution at the dislocations}

In the previous subsection we assumed that there is no dislocation at point $\vec{r}$ at which the probability of the internal stress is determined. One can also ask the probability distribution of the shear stress at the dislocations $P^{c}(\tau)$.

Let assume that the dislocation at which we ask the probability distribution is at the point $\vec{r}_{1}$. The stress at the dislocation is obviously

$$
\tau\left(\vec{r}_{1}\right)=\sum_{i=2}^{N} \tau_{i n d}\left(\vec{r}_{1}-\vec{r}_{i}\right)
$$

The derivation procedure explained above can be repeated for this case, but $f_{N}\left(\vec{r}_{1}, \vec{r}_{2}, \ldots, \vec{r}_{N}\right)$ needs to be replaced with the conditional probability density $f^{c}\left(\vec{r}_{2}, \ldots, \vec{r}_{N} \mid \vec{r}_{1}\right)$.

$f^{c}\left(\vec{r}_{2}, \ldots, \vec{r}_{N} \mid \vec{r}_{1}\right) d \vec{r}_{2} d \vec{r}_{3} . . d \vec{r}_{N}$ is the the probability of finding the 2 nd, 3th, .. Nth dislocation in the $d \vec{r}_{2} d \vec{r}_{3} . . d \vec{r}_{N}$ vicinity of points $\vec{r}_{2}, \vec{r}_{3} . . \vec{r}_{N}$ assuming there is a dislocation at point $\vec{r}_{1}$. One gets that the Fourier transform of $P^{c}(\tau)$ reads as

$$
A^{c}(\vec{r}, q)=\int d^{2} \vec{r}_{2} d^{2} \vec{r}_{3} \ldots d^{2} \vec{r}_{N} f^{c}\left(\vec{r}_{2}, \ldots, \vec{r}_{N} \mid \vec{r}_{1}\right) \prod_{j=2}^{N}\left[1-B\left(\vec{r}_{1}-\vec{r}_{j}, q\right)\right]
$$

The conditional probability $f^{c}\left(\vec{r}_{2}, \ldots, \vec{r}_{N} \mid \vec{r}_{1}\right)$ can be given as

$$
f^{c}\left(\vec{r}_{2}, \ldots, \vec{r}_{N} \mid \vec{r}_{1}\right)=\frac{f_{N}\left(\vec{r}_{1}, \vec{r}_{2}, \ldots, \vec{r}_{N}\right)}{f_{1}\left(\vec{r}_{1}\right)} .
$$

Substituting this into Eq. (4.19) and rewriting it into a power series of $B(\vec{r}, q)$ we obtain

$$
\begin{aligned}
A^{c}\left(\vec{r}_{1}, q\right)= & 1-\int\left[\frac{\rho_{++}\left(\vec{r}_{1}, \vec{r}_{2}\right)}{\rho_{+}\left(\vec{r}_{1}\right)} B\left(\vec{r}_{1}-\vec{r}_{2}, q\right)\right. \\
& \left.+\frac{\rho_{+-}\left(\vec{r}_{1}, \vec{r}_{2}\right)}{\rho_{+}\left(\vec{r}_{1}\right)} B^{*}\left(\vec{r}_{1}-\vec{r}_{2}, q\right)\right] d^{2} \vec{r}_{2}+\ldots
\end{aligned}
$$


where we assumed that the dislocation at point $\vec{r}_{1}$ has positive Burgers vector. (A similar expression holds for dislocations with negative Burgers vector.)

\subsection{The mean values of distributions $P(\tau)$ and $P^{c}(\tau)$}

Since dislocations form strongly inhomogeneous distributions the explicit form of $P(\tau)$ and $P^{c}(\tau)$ cannot be determined analytically. Nevertheless, analytical results can be obtained for some of their properties [71, 73].

An important characteristic value of a distribution function $P(x)$ is its mean value $<x>=\int x P(x) d x$. As it is well known the mean value can be determined from the $A(q)$ Fourier transform of the distribution function by the relation

$$
<x>=\left.\frac{i}{A(0)} \frac{d A(q)}{d q}\right|_{q=0}
$$

Applying the above expression for Eqs. $(4.17,4.21)$ we find that the mean value of the internal stress at a point where there is no dislocations is

$$
\begin{aligned}
<\tau(\vec{r})> & =-i \int\left[\left.\rho_{+}(\vec{r}) \frac{d B}{d q}\right|_{q=0}+\left.\rho_{-}(\vec{r}) \frac{d B^{*}}{d q}\right|_{q=0}\right] d \vec{r}_{1} \\
& =\int \kappa(\vec{r}) \tau_{\text {ind }}\left(\vec{r}-\vec{r}_{1}\right) d \vec{r}_{1} .
\end{aligned}
$$

If we compare this with expression (3.40) we can see that $\langle\tau(\vec{r})\rangle$ is the self-consistent field introduced earlier.

On the other hand, from Eq. (4.21) the mean value of shear stress at a dislocation is

$$
\begin{aligned}
<\tau_{+}\left(\vec{r}_{1}\right)> & =-i \int\left[\left.\frac{\rho_{++}\left(\vec{r}_{1}, \vec{r}_{2}\right)}{\rho_{+}\left(\vec{r}_{1}\right)} \frac{d B}{d q}\right|_{q=0}+\left.\frac{\rho_{+-}\left(\vec{r}_{1}, \vec{r}_{2}\right)}{\rho_{+}\left(\vec{r}_{1}\right)} \frac{d B^{*}}{d q}\right|_{q=0}\right] d \vec{r}_{2} \\
& =\frac{1}{\rho\left(\vec{r}_{1}\right)} \int\left[\rho_{++}\left(\vec{r}_{1}, \vec{r}_{2}\right)-\rho_{+-}\left(\vec{r}_{1}, \vec{r}_{2}\right)\right] \tau_{\text {ind }}\left(\vec{r}-\vec{r}_{1}\right) d \vec{r}_{2}
\end{aligned}
$$

(The subscript "+" in $<\tau_{+}\left(\vec{r}_{1}\right)>$ indicates that it corresponds to a dislocation with positive sign.)

If we compare the above expression with the third terms in Eqs. $(3.28,3.29)$ we can see that Eqs. $(3.28,3.29)$ can be rewritten as

$$
\begin{aligned}
& \frac{\partial \rho_{+}\left(\vec{r}_{1}, t\right)}{\partial t}+\vec{b} \frac{\partial}{\partial \vec{r}_{1}}\left\{\rho_{+}\left(\vec{r}_{1}, t\right)\left[\tau_{\text {ext }}+<\tau_{+}\left(\vec{r}_{1}\right)>\right]\right\}=0 \\
& \frac{\partial \rho_{-}\left(\vec{r}_{1}, t\right)}{\partial t}-\vec{b} \frac{\partial}{\partial \vec{r}_{1}}\left\{\rho_{-}\left(\vec{r}_{1}, t\right)\left[\tau_{\text {ext }}+<\tau_{-}\left(\vec{r}_{1}\right)>\right]\right\}=0
\end{aligned}
$$

giving a new physical interpretation of the form of the dislocation evolution equations obtained. 


\subsection{Asymptotic properties of the stress distribution function}

According to the investigations of Groma and Bakó [71] for small enough Fourier parameter $q$ the Fourier transform of $P(\tau)$ reads as (for the derivation see [71]):

$$
A_{N}(\vec{r}, q)=\exp \left\{i<\tau(\vec{r})>q+C_{d} \rho(\vec{r}) q^{2} \ln \frac{q}{R_{e f f}}+\ldots\right\},
$$

where $R_{e f f}$ is an effective correlation length determined by the correlation functions $d_{++}$, $d_{--}, d_{+-}, d_{-+}$, and

$$
C_{d}=\frac{b^{2}}{2} \int_{0}^{2 \pi} r^{2} \tau_{i n d}^{2}(r, \varphi) d \varphi
$$

For edge dislocations in isotropic medium

$$
C_{d}=\frac{(\mu b)^{2}}{8 \pi^{2}(1-\nu)^{2}} \int_{0}^{2 \pi} \cos ^{2}(\varphi) \cos ^{2}(2 \varphi) d \varphi=\frac{(\mu b)^{2}}{16 \pi(1-\nu)^{2}} .
$$

It has to be mentioned that if the dislocation system consists of more than one type of dislocations, Eq. (4.27) remains valid. The only difference is that in this case $R_{\text {eff }}$ and $C_{d}$ are the appropriate weighted average of the $R_{\text {eff }}^{i}$ and $C_{d}^{i}$ values corresponding to the dislocations with Burgers vector $\vec{b}^{i}$.

One can obtain (see [71]) that Eq. (4.27) follows that the tail of probability distribution function decays as

$$
P(\tau)=C_{d} \rho(\vec{r}) \frac{1}{\tau^{3}} .
$$

A remarkable feature of the above expression is that it is independent from the dislocationdislocation correlations. In other words it does not depend on the microscopic arrangement of the dislocations. Certainly the stress value from which the distribution function follows the above asymptotic expression depends on dislocation-dislocation correlation. To demonstrate this, the internal stress distribution was numerically determined on a system of 1000 randomly distributed dislocation dipoles (seen Figure 19). As it can be seen the asymptotic curve describes extremely well the tail part of the internal stress distribution obtained numerically. It has to be mentioned that the asymptotic curve is not fitted, $C_{d}$ is determined according to Eq. (4.29).

Recent investigations revealed [73] that for a dipole system the centre part of the distribution is Lorentzian:

$$
P(\tau)=\frac{D_{d} \rho}{\pi} \frac{1}{\tau^{2}+(D \rho)^{2}}
$$

where the actual value of $D$ can only be determined numerically. For edge dislocation dipoles with $45^{0}$ between the polarisation and the Burgers vectors

$$
D_{d}=\frac{b \mu}{2 \pi(1-\nu)} 3.55|\vec{p}|
$$




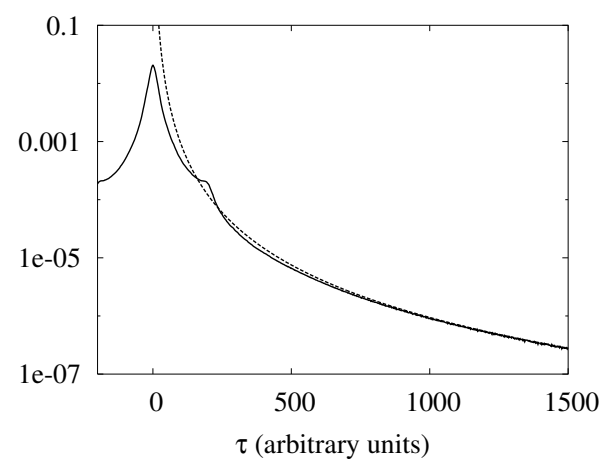

Figure 19. $P(\tau)$ obtained numerically on a randomly distributed dipole system (full line) with the asymptotic curve given by Eq. (4.30) (dotted line).

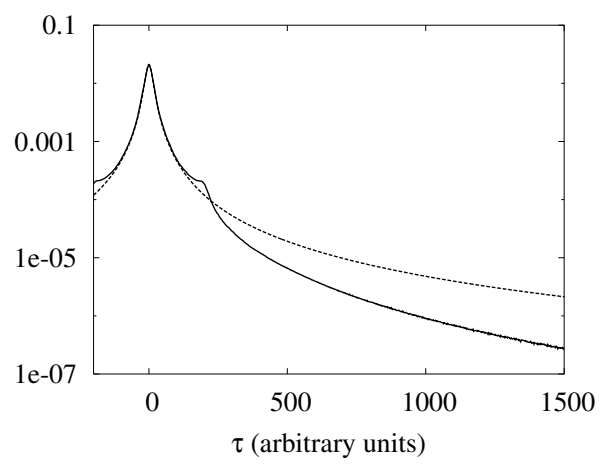

Figure 20. $P(\tau)$ obtained numerically on a randomly distributed dipole system (full line) with the Lorentzian given by Eq. (4.31) (dotted line).

As it can be seen in Figure 20 the centre part of $P(\tau)$ is well described by the Lorentzian given by Eq. (4.31).

\section{Acknowledgement}

During the past years the author had the pleasure to work with Botond Bakó, Ferenc Csikor, Erik van der Giessen, Géza Györgyi, Péter Dusán Ispánovity, Ferenc Székely, Serge Yefimov and Michael Zaiser. Most of the results presented in the paper were obtained together with them. Their outstanding scientific contributions and friendships are gratefully acknowledged.

Financial supports of the Hungarian Scientific Research Fund (OTKA) under contract number K 67778 and the European Community's Human Potential Programme under contracts HPRN-CT-2002-00198 [DEFINO] and MRTN-CT-2003-504634 [SizeDe- 
pEn] are also acknowledged.

\section{Bibliography}

[1] I. Kovács and L. Zsoldos, Dislocations and Plastic Deformation, Pergamon Press, London (1973).

[2] Basinski S.J. and Basinski Z.S., Dislocations in Solids, ed. by F.R.N. Nabarro, NorthHolland, Amsterdam, 1979

[3] Hirsh JP. Lothe J. Theory of dislocations, John Wiley and Sons, 1982

[4] Mughrabi H., Ungár T., Kienle W., M. Wilkens, Phil. Mag. 53, 793 (1986)

[5] Mughrabi H., Ackerman F., Herz K., in J. Fang (editor), Fatigue Mechanisms, Proc. of ASTM-NBS-NSF Symposium, ASTM-STP 675, 1979, p. 69.

[6] Kuhlmann-Wilsdorf D., van der Merwe J., Mat. Sci. Eng. 35, 79 (1982)

[7] Bassim M., Jesser W., Kuhlmann-Wilsdorf D., Wilsdorf H., Mat. Sci. Eng. 81, $122(1986)$

[8] Kuhlmann-Wilsdorf D., Mat. Sci. Eng. A 113, 1 (1989)

[9] Holt D., Journal Appl. Phys. 41, 3179 (1970)

[10] Walgraef D., Aifantis E., J. Appl. Phys. 15, 688 (1985)

[11] Aifantis E., Mat. Sci. Eng. 81, 563 (1986)

[12] Walgraef D., Solid State Phenomena, 3-4, 77 (1988)

[13] Kratochvil J., Scripta Metall. Mater. 24, 891 (1990)

[14] Kratochvil J., Scripta Metall. Mater. 24, 1225 (1990)

[15] Kratochvil J., and Saxlova M., Scripta Met. Mater. 26, 113 (1993)

[16] Hähner P., Bay K., Zaiser M., Phys. Rev. Lett. 81, 2470 (1998)

[17] Zaiser M., Bay K., Hähner P., Acta mater. 47, 2463 (1999)

[18] Székely F., Groma I., Lendvai J., Mat. Sci. Eng. A 309, 352 (2001)

[19] Székely F., Groma I., Lendvai J., Scripta Mater 45, 55 (2001)

[20] Fleck N.A., Mller G.M., Ashby M.F. and Hutchinson J.W., Acta Mater. 42, 475 (1994)

[21] McElhaney K.W., Vlassah JJ. and Nix W.D., J. Math. Res. 13, 1300 (1998)

[22] Aifantis E.C., J. Eng. Mater. Technol. 106, 326 (1984)

[23] Aifantis E.C., Int. J. Plast. 2, 211 (1987)

[24] Aifantis E.C., J. Mech. Behav. Mater. 5, 335 (1994)

[25] Fleck N.A. and Hutchinson J.W., J. Mech. Phys. Solids, 49, 2245 (2001)

[26] Gurtin M.E., J. Mech. Phys. Solids, 50, 5 (2002)

[27] Svendsen B., J. Mech. Phys. Solids, 50, 1297 (2002)

[28] Nicola L., van der Giessen E. and Needleman A., Journal of Applied Physics 93, $5920(2003)$

[29] Kröner E., Z. Phys. 151, 504 (1958)

[30] E. Kröner, Continuum Theory of Defects, Physics of Defects, Eds. R. Balian et.all, North Holland, Amsterdam (1981).

[31] L.D. Landau, E.M. Lifsitz, Theory of Elasticity, Course of Theoretical Physics, vol 7, Pergamon Press, Oxford (1986) 
[32] Kosevich A.M. in Dislocations in Solids, ed. by F.R.N. Nabarro, North-Holland, Amsterdam, 1979

[33] Nye J.F. Acta. Mat. 1, 153 (1953)

[34] Burgers J.M. Proc. Kon. Nederl. Akad. Wetensch. 42, 293 and 378 (1939)

[35] Devincre B., and Condat M., Acta Metall. Mater. 40, 2629 (1992)

[36] Fivel M., Gosling T.,and Canova, Modell. Simul. Mater. Sci. Eng. 4, 581 (1996)

[37] Huang J.M., and Ghoniem N.M., Model. Simul. Mater. Sci. 11, 21 (2003)

[38] Singh B.N., Ghoniem N.M., and Trinkaus H. J. Nucl. Mater. 307, 159 (2002)

[39] Huang H.M., and Ghoniem N.M. Comp. Mater. Sci. 23, 225 (2002)

[40] Walgraef D., and Ghoniem N.M. Phys. Rev. B 65, art. no. 155304 (2002)

[41] Madec R., Devincre B., and Kubin L.P., Phys. Rev. Lett. 89, 255508 (2002)

[42] Madec R., Devincre B., and Kubin L.P. Scripta Mater 47, 689 (2002)

[43] Lemarchand C., Chaboche J.L., and Devincre B,. J. Phys IV 9, 271 (1999)

[44] Bulatov V., Abraham F.F., Kubin L., Devincre B., and Yip S., Nature 391, 669 (1998)

[45] Cai W., Bulatov V.V., Chang J., Li J. and Yip S., Phil. Mag. A, 81, 1257 (2001).

[46] Lepinoux J., and Kubin L., Scripta Met. Mat. 21, 833 (1987)

[47] Gulluoglu A., and Hartly C., Modell. Simul. Mater. Sci. Eng. 1, 383 (1993)

[48] Gullouglu A., Srolovity D., LeSar R., and Lomdahl P., Scripta Met. 23, 1347 (1989)

[49] Ghoniem N., and Amodeo R., Phys. Rev. B 41, 6968 (1989)

[50] Groma I. and Pawley G.S., Philos. Mag. A 671459 (1993)

[51] Groma I. and Pawley G.S., Mat. Sci. Eng. A 164, 306 (1993)

[52] Van der Giessen E., and Needleman A., Model. Simul. Mater. Sci. Eng. 3, 689 (1995)

[53] Cleveringa H. H. M., V. der Giessen E., and Needleman A., Acta mater. 45, 3163 (1997)

[54] Cleveringa, H.H.M., Van der Giessen, E., and Needleman, Acta Mater. 45, 3163 (1997)

[55] Cleveringa, H.H.M., Van der Giessen, E. and Needleman, A., International Journal of Plasticity 15, 837 (1999)

[56] Cleveringa, H.H.M., Van der Giessen, E. and Needleman, A., Phil. Mag. A79, 863 (1999)

[57] A. Benzerga, Y. Brechet, A. Needleman and E. Van der Giessen Model. Simul. Mat. Sci. Eng. 12, 159 (2004)

[58] Groma I., Phys. Rev. B 56, 5807 (1997)

[59] Groma I. and Balogh P., Acta Mater. 47, 3647 (1999)

[60] Buchinger L. and Stanzl S., Phil. Mag. A50, 275 (1984)

[61] Zaiser M., Miguel M.C., Groma I., Phys Rev B 64, 224102 (2001)

[62] Groma I., Csikor FF., Zaiser M., Acta Mater. 51, 1271 (2003)

[63] Cleveringa, H.H.M., Van der Giessen, E. and Needleman, A., J. Physique IV, 83, 92 (1998)

[64] Yefimov S., Groma I. and van der Giessen E. J. de Physique IV 11, 103 (2001)

[65] S. Yefimov, I. Groma and E. van der Giessen J. Mech. and Phys. Solids 52, 279 (2004) 
[66] Yefimov S. and van der Giessen E European Journal of Mechanics A/Solids 24, 183 (2005)

[67] Yefimov S. and van der Giessen E. Int. J. of Solids Struct. 42, 3375 (2005)

[68] El-Azab A., Phys. Rev. B, 61, 11956, (2000)

[69] Hochrainer T. and Zaiser M., private communication

[70] Kratochvíl J. and Sedláček R., Phys. Rev. B, 67, 094105 (2003)

[71] Groma I. and Bak B., Phys. Rev. B 58, 2969 (1998)

[72] Chandrasekhar S., Rev. Modern Phys. 15, 8 (1943)

[73] Csikor F.F. Groma I. Phys. Rev. B 70, 064106 (2004)

[74] Groma I.,Phys. Rev. B 57, 7535 (1998) 